\title{
DN interaction from meson exchange
}

\author{
J. Haidenbauer ${ }^{1,2, a}$, G. Krein ${ }^{3}$, U.-G. Meißner ${ }^{1,2,4}$, and L. Tolos ${ }^{5,6}$ \\ 1 Institut für Kernphysik and Jülich Center for Hadron Physics, Forschungszentrum Jülich, D-52425 Jülich, Germany \\ 2 Institute for Advanced Simulation, Forschungszentrum Jülich, D-52425 Jülich, Germany \\ 3 Instituto de Física Teórica, Universidade Estadual Paulista, Rua Dr. Bento Teobaldo Ferraz, 271 - 01140-070 São Paulo, SP, \\ Brazil \\ 4 Helmholtz-Institut für Strahlen- und Kernphysik (Theorie) and Bethe Center for Theoretical Physics, Universität Bonn, \\ Nußallee 14-16, D-53115 Bonn, Germany \\ 5 Theory Group, KVI, Zernikelaan 25, NL-9747AA Groningen, The Netherlands \\ 6 Instituto de Ciencias del Espacio (IEEC/CSIC), Campus Universitat Autonoma de Barcelona, Facultat de Ciencies, Torre \\ C5, E-08193 Bellaterra (Barcelona), Spain
}

Received: 23 August 2010 / Revised: 15 November 2010

Published online: 3 February 2011

(c) The Author(s) 2011. This article is published with open access at Springerlink.com

Communicated by A. Ramos

\begin{abstract}
A model of the $D N$ interaction is presented which is developed in close analogy to the mesonexchange $\bar{K} N$ potential of the Jülich group utilizing $S U(4)$ symmetry constraints. The main ingredients of the interaction are provided by vector meson $(\rho, \omega)$ exchange and higher-order box diagrams involving $D^{*} N, D \Delta$, and $D^{*} \Delta$ intermediate states. The coupling of $D N$ to the $\pi \Lambda_{c}$ and $\pi \Sigma_{c}$ channels is taken into account. The interaction model generates the $\Lambda_{c}(2595)$-resonance dynamically as a $D N$ quasi-bound state. Results for $D N$ total and differential cross sections are presented and compared with predictions of two interaction models that are based on the leading-order Weinberg-Tomozawa term. Some features of the $\Lambda_{c}(2595)$-resonance are discussed and the role of the near-by $\pi \Sigma_{c}$ threshold is emphasized. Selected predictions of the orginal $\bar{K} N$ model are reported too. Specifically, it is pointed out that the model generates two poles in the partial wave corresponding to the $\Lambda(1405)$-resonance.
\end{abstract}

\section{Introduction}

The study of the low-energy interactions of open charm $D$-mesons with nucleons is challenging for several reasons. The complete lack of experimental data on the interaction makes it very difficult to constrain models. Reliable models are very important for guiding planned experiments by the PANDA [1] and CBM [2] Collaborations at the future FAIR facility at Darmstadt [3]. Estimates for the magnitudes of cross sections are required for the design of detectors and of efficient data acquisition systems. As emphasized in recent publications $[4,5]$, one way to make progress in such a situation is to build models guided by analogies with other similar processes, by the use of symmetry arguments and of different dynamical degrees of freedom.

Physics motivations for studying the interaction of $D$ mesons with nucleons are abundant. Amongst the most exciting ones is the possibility of studying chiral symmetry in matter. The chiral properties of the light quarks composing $D$-mesons are sensitive to temperature and den-

\footnotetext{
a e-mail: j.haidenbauer@fz-juelich.de
}

sity; one expects to learn about manifestations of such a sensitivity through the detection of changes in the interaction of these mesons with nucleons in the medium as compared to the corresponding interaction in free space. Also, studies of $J / \psi$ dissociation in matter, since long time [6] suggested as a possible signature for the formation of a quark-gluon plasma, require a good knowledge of the interaction of $D$-mesons with ordinary hadrons [7] in order to differentiate different scenarios and models in this area. Another exciting perspective is the possibility of the formation of $D$-mesic nuclei $[8,9]$ and of exotic nuclear bound states like $J / \psi$ binding to nuclei $[10,11]$. In this latter case in particular, the interaction of $D$-mesons with ordinary matter plays a fundamental role in the properties of such exotic states $[12,13]$.

In a recent paper we examined the possibility to extract information about the $D N$ and $\bar{D} N$ interactions from the $\bar{p} d \rightarrow D^{0} D^{-} p$ reaction [5]. The scattering amplitudes for $D N$ and $\bar{D} N$ employed in this exploratory study were generated from interaction models that were constructed along the line of the $\bar{K} N$ and $K N$ mesonexchange potentials developed by the Jülich group some time ago $[14-16]$. While the $\bar{D} N$ interaction has been 
described in some detail in ref. [4] this has not been done so far for the $D N$ model. With the present paper we want to remedy this situation.

The $D N$ interaction is derived in close analogy to the meson-exchange $\bar{K} N$ model of the Jülich group [14], utilizing as a working hypothesis $S U(4)$ symmetry constraints, and by exploiting also the close connection between the $D N$ and $\bar{D} N$ systems due to $G$-parity conservation. In particular, the $D N$ potential is obtained by substituting the one-boson-exchange $(\sigma, \rho, \omega)$ contributions, but also the box diagrams involving $\bar{K}^{*} N, \bar{K} \Delta$, and $\bar{K}^{*} \Delta$ intermediate states, of the original $\bar{K} N$ model of the Jülich group by the corresponding contributions to the $D N$ interaction. Of course, we know that $S U(4)$ symmetry is strongly broken, as is reflected in the mass differences between the strangeness and the charm sectors, for mesons as well as for baryons. However, as already argued in ref. [4], we expect that the dynamics in the $D N(\bar{D} N)$ and $\bar{K} N$ $(K N)$ systems should be governed predominantly by the same "long-range" physics, i.e. by the exchange of ordinary (vector and possibly scalar) mesons. Thus, in both systems the dynamics should involve primarily the upand down-quarks, whereas the heavier quarks (the $s$ and $c$ quarks, respectively) behave more or less like spectators and contribute predominantly to the static properties of the mesons and baryons. Therefore, the assumption of $S U(4)$ symmetry for the dynamics seems to be not completely implausible.

In any case, invoking $S U(4)$ symmetry for the $D N$ interaction involves larger uncertainties than for $\bar{D} N$. For the former, like in the analogous $\bar{K} N$ system, there are couplings to several other channels which are already open near the $D N$ threshold $\left(\pi \Lambda_{c}, \pi \Sigma_{c}\right)$ or open not far from the threshold $\left(\eta \Lambda_{c}\right)$. The coupling to those channels should play an important role for the dynamics of the $D N$ system - as is the case in the corresponding $\bar{K} N$ systemand, thus, will have an impact on the results, at least on the quantitative level. Specifically, the transitions from $D N$ to those channels involve the exchange of charmed mesons, for example the $D^{*}(2010)$, where the range arguments given above no longer hold, and where the coupling constants and associated vertex form factors, required in any meson-exchange model, are difficult to constrain.

Another open issue is the relevance of heavy-quark symmetry (HQS). This symmetry which is an exact symmetry of the underlying QCD Lagrangian in the limit of large quark masses $[17,18]$, connects the coupling strengths of specific spin doublets such as the $D$ and $D^{*}$ mesons or the $\Sigma_{c}$ and $\Sigma_{c}^{*}$ baryons $[19,20]$. While the coupling constants that are used in our $D N$ (and also in our $\bar{D} N)$ model comply with the restrictions imposed by HQS, simply because $S U(6)$ symmetry was used in deriving the Jülich $\bar{K} N$ and $K N$ models, HQS is not implemented fully consistently in our $D N$ and $\bar{D} N$ models.

In 1993 a first evidence for the $\Lambda_{c}(2595)$-resonance was reported by the CLEO Collaboration [21] and subsequently confirmed by several other experiments [22-24]. Nowadays it is generally accepted that this resonance is the charmed counterpart of the $\Lambda(1405)$ [25]. In the Jülich $\bar{K} N$ potential model [14] the latter state is generated dy- namically. It appears as a $\bar{K} N$ quasi-bound state and is produced by the strongly attractive interaction due to the combined effect of $\omega, \rho$ and scalar-meson exchanges, which add up coherently in the $\bar{K} N$ channel. When extending the Jülich meson-exchange model to the charm sector via $S U(4)$ symmetry one expects likewise the appearance of a quasi-bound state, namely in the $D N$ channel. Thus, one can actually utilize the experimentally known mass of the $\Lambda_{c}(2595)$-resonance as an additional constraint for fixing parameters of the $D N$ interaction.

Indeed such a strategy was already followed in recent studies of the $\bar{K} N$ and $D N$ interaction within chiral unitary (and related) approaches, where likewise the 1 (1405)resonance but also states in the $D N$ system are generated dynamically [26-31]. In those approaches the strong attraction is also provided by vector-meson exchange [27, 31 , by the Weinberg-Tomazawa (WT) term $[26,28,29]$, or by an extension of the WT interaction to an $S U(8)$ spin-flavor scheme [30]. In refs. [28-33] the authors argued that a state occurring in the $S_{01}$ channel of the charm $C=1$ sector should be identified with the $I=0$ resonance $\Lambda_{c}(2595)$. (Throughout we use the standard spectroscopical nomenclature $L_{I 2 J}$, with $L$ denoting the orbital angular momentum, $I$ the isospin and $J$ the total angular momentum of the two-particle system.) Note, however, that some of those works differ with regard to the nature of the $\Lambda_{c}(2595)$, i.e. in the dominant meson-baryon component of this state. For example, in the $S U(8)$ spin-flavor scheme of ref. [30] the $\Lambda_{c}(2595)$ becomes predominantly a $D^{*} N$ quasi-bound state - though still with important additional binding effects from the $D N$ channel.

We take the opportunity to present here also some results of the original Jülich $\bar{K} N$ model [14]. Over the last years there has been increasing interest in the properties of the $\bar{K} N$ interaction close to the threshold and the near-by $\Lambda(1405)$-resonance, resulting in a vast amount of pertinent publications. Indeed, there is still a controversy about the actual value for the strong-interaction energy shift of kaonic hydrogen and there are ongoing debates about issues like a possible double-pole structure of the $\Lambda(1405)$ or deeply-bound kaonic states (for recent overviews, see refs. [34-37]). The initial publication of the Jülich group [14] focussed on a detailed account of the ingredients of the $\bar{K} N$ interaction model and on the description of scattering data available at that time. Results of the Jülich model for quantities that are relevant for the issues mentioned above were not given. This will be remedied by the present paper. Specifically, we provide here the $\bar{K} N$ scattering length and we determine the pole position corresponding to the $\Lambda(1405)$-resonance. For the latter it turns out that also the $\bar{K} N$ model of the Jülich group predicts the existence of two poles in the corresponding $S_{01}$ $\bar{K} N$ partial wave.

The paper is organized in the following way: in sect. 2 a brief description of the ingredients of the $D N$ model is given. The interaction Lagrangians, which are used to derive the meson-baryon potential, are summarized in the appendix. In sect. 3 selected results for the Jülich $\bar{K} N$ model are presented. Results for $D N$ scattering are presented in sect. 4 . Besides the scattering lengths and the 

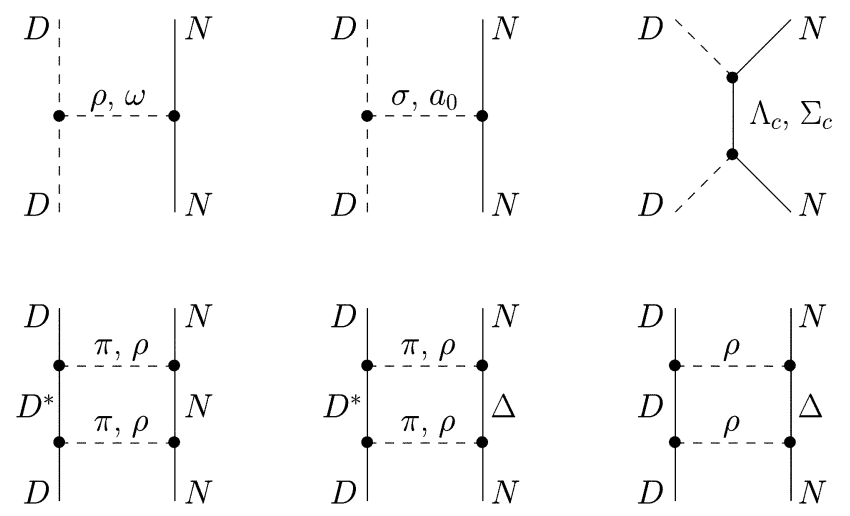

Fig. 1. Meson-exchange contributions included in the direct $D N$ interaction.

pole positions corresponding to the $\Lambda_{c}(2595)$-resonance, also some predictions for total and differential cross sections are given. Furthermore, we compare our results with those obtained from $D N$ interactions that were derived from the leading-order WT contact term, assuming either $S U(4)$ symmetry [28] or even $S U(8)$ symmetry [30]. Considering those results allows us to explore the model dependence of the predictions for $D N$ scattering observables. Section 5 is dedicated to the $\Lambda_{c}(2595)$-resonance and focusses on the consequences of the fact that the position of this resonance coincides practically with the $\pi \Sigma_{c}$ threshold. In particular, we present results for the $\pi \Sigma_{c}$ invariantmass spectrum which allows us to discuss the subtle effects of the slightly different thresholds of the $\pi^{+} \Sigma_{c}^{0}, \pi^{0} \Sigma_{c}^{+}$, and $\pi^{-} \Sigma_{c}^{++}$channels on the various invariant-mass distributions. Also here a comparison with results based on the $S U(4)$ (and $S U(8)$ ) WT approach is made. Furthermore, we discuss similarities and differences between the $\Lambda(1405)$ and the $\Lambda_{c}(2595)$ as dynamically generated states. The paper ends with a short summary.

\section{Coupled-channel DN model in the meson-exchange framework}

The $D N$ interaction employed in the present study is constructed in close analogy to the meson-exchange $\bar{K} N$ model of the Jülich group [14] utilizing $S U(4)$ symmetry constraints, as well as by exploiting the close connection between the $D N$ and $\bar{D} N$ systems due to $G$-parity conservation. Specifically, we use the latter constraint to fix the contributions to the direct $D N$ interaction potential while the former one provides the transitions to and interactions in channels that can couple to the $D N$ system. The main ingredients of the $D N \rightarrow D N$ interaction are provided by vector meson $(\rho, \omega)$ exchange and higher-order box diagrams involving $D^{*} N, D \Delta$, and $D^{*} \Delta$ intermediate states, see fig. 1.

The original $\bar{K} N$ and $K N$ models of the Jülich group include besides the exchange of the standard mesons also an additional phenomenological (extremely short-ranged) repulsive contribution, a " $\sigma_{\text {rep }}$ ", with a mass of about
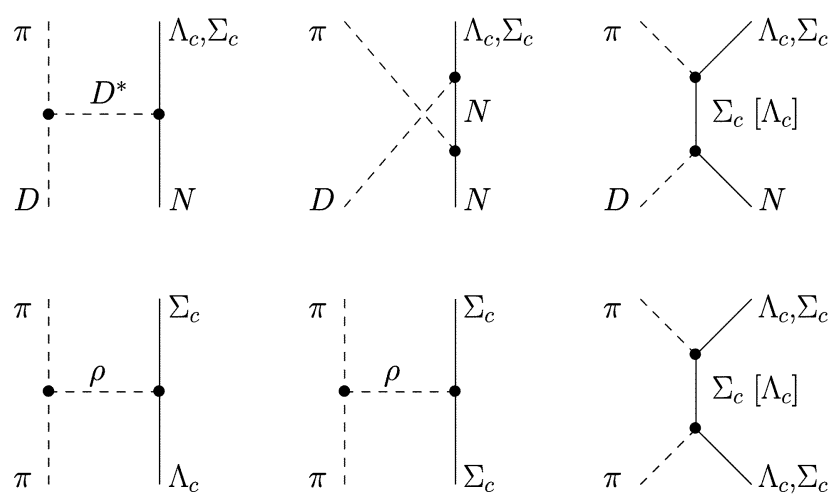

Fig. 2. Meson-exchange contributions included in the $D N \rightarrow$ $\pi \Lambda_{c}, \pi \Sigma_{c}$ transition potentials and in the $\pi \Lambda_{c}, \pi \Sigma_{c} \rightarrow \pi \Lambda_{c}$, $\pi \Sigma_{c}$ interactions.

1.2 GeV [14,16]. This contribution was introduced ad hoc in order to achieve a simultaneous description of the empirical $K N S$ - and $P$-wave phase shifts $[15,16]$. Evidently, due to its phenomenological nature it remains unclear how that contribution should be treated when going over to the $\bar{D} N$ and $D N$ systems. This difficulty was circumvented in the construction of the $\bar{D} N$ interaction [4] by resorting to a recent study of the $K N$ interaction [38] which provided evidence that a significant part of that short-ranged repulsion required in the original Jülich model could be due to genuine quark-gluon exchange processes. Indeed, considering such quark-gluon mechanisms together with conventional $a_{0}(980)$-meson exchange instead of that from " $\sigma_{\text {rep }}$ " a comparable if not superior description of $K N$ scattering could be achieved [38]. From this model the $\bar{D} N$ interaction [4] could be derived in a straightforward way under the assumption of $S U(4)$ symmetry. Furthermore, the extension to the $D N$ system is possible too. Note, however, that the short-ranged quark-gluon processes, that contribute to the $\bar{D} N$ interaction [4], are absent here because the quark-exchange mechanism cannot occur in the $D N$ interaction due to the different quark structure of the $D$-meson as compared to $\bar{D}$.

As far as the coupling to other channels is concerned, we follow here the arguments of ref. [14] and take into account only the channels $\pi \Lambda_{c}(2285)$ and $\pi \Sigma_{c}(2455)$. Furthermore, we restrict ourselves to vector-meson exchange and we do not consider any higher-order diagrams in those channels. Pole diagrams due to the $\Lambda_{c}(2285)$ and $\Sigma_{c}(2455)$ intermediate states are, however, consistently included in all channels. The various contributions to the $D N \rightarrow \pi \Lambda_{c}, \pi \Sigma_{c}$ transition potentials and to the $\pi \Lambda_{c}, \pi \Sigma_{c} \rightarrow \pi \Lambda_{c}, \pi \Sigma_{c}$ interaction, taken into account in the present model, are depicted in fig. 2 .

HQS would require to include further channels in our model. Specifically the $D N$ and $D^{*} N$ channels and the corresponding interactions should be treated on equal footing. While both channels are indeed taken into account in our model, the direct interaction in the $D^{*} N$ case has been neglected in analogy to what has been done for $\bar{K}^{*} N$ in the Jülich $\bar{K} N$ model [14]. Thus, being faced 
with the decision whether we should maintain the close relation with the original Jülich $\bar{K} N$ model or impose fully HQS we chose the former option. Still we would like to point out that the $D N$ interaction and the $D N \rightarrow D^{*} N$ transition potential, as mediated by $\rho$ exchange, are consistent with HQS due to the $S U(6)$ symmetry that was adopted in the construction of the Jülich $\bar{K} N$ and $K N$ models.

The interaction Lagrangians, which are used to derive the meson-baryon potentials for the different channels, are summarized in the appendix. Based on the resulting interaction potentials $\mathcal{V}_{i j}\left(i, j=D N, \pi \Lambda_{c}, \pi \Sigma_{c}\right)$ the corresponding reaction amplitudes $\mathcal{T}_{i j}$ are obtained by solving a coupled-channel Lippmann-Schwinger-type scattering equation within the framework of time-ordered perturbation theory,

$$
\mathcal{T}_{i j}=\mathcal{V}_{i j}+\sum_{k} \mathcal{V}_{i k} \mathcal{G}_{k}^{0} \mathcal{T}_{k j}
$$

from which we calculate the observables in the standard way [15].

The assumed $S U(4)$ symmetry and the connection with the $\bar{K} N$ model, respectively, allows us to fix most of the parameters - the coupling constants and the cutoff masses at the vertex form factors of the occurring meson-meson-meson and meson-baryon-baryon vertices, cf. ref. [14]. A list with the specific values of the pertinent parameters can be found in the appendix.

When solving the coupled-channel Lippmann-Schwinger equation with this interaction model we observe that two narrow states are generated dynamically below the $D N$ threshold, one in the $S_{01}$ partial wave and the other one in the $S_{11}$ phase. In view of the close analogy between our $D N$ model and the corresponding $\bar{K} N$ interaction [14] this is not too surprising, because the latter also yields a quasi-bound state in the $S_{01}$ channel which is associated with the $1(1405)$-resonance. The bound states in both $\bar{K} N$ and $D N$ are generated by the strongly attractive interaction due to the combined effect of $\omega, \rho$ and scalar-meson exchanges, which add up coherently in specific channels.

Following the arguments in refs. [28-30,32,33] we identify the narrow state occurring in the $S_{01}$ channel with the $I=0$ resonance $\Lambda_{c}(2595)$. Furthermore, we identify the state we get in the $S_{11}$ channel with the $I=1$ resonance $\Sigma_{c}(2800)$ [25]. (For a different scenario, see [39].) In order to make sure that the $D N$ model incorporates the above features also quantitatively, the contributions of the scalar mesons to the $D N$ interaction are fine-tuned so that the position of those states generated by the model coincide with the values given by the Particle Data Group [25]. This could be achieved by a moderate change in the coupling constants of the $\sigma$-meson (from 1 to 2.6 ) and the $a_{0}$-meson (from -2.6 to -4.8 ), cf. table 3 in the appendix. We would like to stress that anyhow the application of $S U(4)$ symmetry (and even $S U(3)$ symmetry) to the scalar-meson sector is problematic, as discussed in ref. [4]. In the present paper we will show results for the latter interaction which we consider as our basic model. However, we present also
$D N$ cross sections based on the parameter set that follows directly from the $\bar{K} N$ and $K N$ studies $[15,16,38]$ by assuming $S U(4)$ symmetry. Those results may be considered as a measure for the uncertainty in our model predictions for $D N$.

With regard to the $D^{*}(2010)$ exchange that contributes to the $D N \rightarrow \pi \Lambda_{c}$ and $D N \rightarrow \pi \Sigma_{c}$ transition potentials (cf. fig. 2) it should be said that the corresponding form factors cannot be inferred from the $\bar{K} N$ model [14] via $S U(4)$ arguments. We fixed the relevant cut-off masses somewhat arbitrarily to be about $1 \mathrm{GeV}$ larger than the exchange mass. Anyway, variations in those cut-off masses have very little influence on the results in the $D N$ channel. Moreover, within the spirit of the basic model such variations can be easily compensated by re-adjusting the coupling constants in the scalar sector so that again the masses of the $\Lambda_{c}(2595)$ and $\Sigma_{c}(2800)$ resonances are reproduced, as discussed above. But the width of those states are certainly sensitive to the values used for those cut-off masses.

\section{Selected results of the Jülich $\bar{K} N$ model}

Before discussing the results of the Jülich $D N$ model in detail, let us first present some selected results of the corresponding $\bar{K} N$ model. The general scheme and also the explicit expressions for the various contributions to the $\bar{K} N$ interaction potential of the Jülich group are described in detail in ref. [14]. In the original publication of the model, results for total cross sections of the reaction channels $K^{-} p \rightarrow K^{-} p, K^{-} p \rightarrow K^{0} n, K^{-} p \rightarrow \pi^{0} \Lambda, K^{-} p \rightarrow \pi^{0} \Sigma^{0}$, $K^{-} p \rightarrow \pi^{-} \Sigma^{+}$, and $K^{-} p \rightarrow \pi^{+} \Sigma^{-}$were presented and compared with available data. One can see [14] that the model yields a quite satisfactory description of the available experimental information up to $\bar{K}$ laboratory momenta of $p_{l a b} \approx 300 \mathrm{MeV} / c$. In the present paper we refrain from showing those results again but we want to focus on additional predictions of the model that have not been included in ref. [14]. First this concerns the behaviour close to the $\bar{K} N$ threshold. The original Jülich potential is derived under the assumption of isospin symmetry and the reaction amplitudes were obtained by solving a LippmannSchwinger-type scattering equation (1) in the isospin basis using averaged masses of the involved baryons and mesons. The corresponding $S$-wave scattering lengths $a$ for $I=0$, $I=1$, and for $K^{-} p\left(a_{K^{-} p}=\left(a_{0}+a_{1}\right) / 2\right)$ are summarized in table 1 . In order to enable a detailed comparison with available empirical information in the threshold region, now we also performed a calculation in the particle basis. Using the physical masses of the baryons and mesons allows us to take into account the isospin-breaking effects generated by the known mass splittings within the involved isospin multiplets, and specifically between the $K^{-}$and $\bar{K}^{0}$ masses. The $K^{-} p$ scattering length calculated in this way is also given in table 1 . It differs significantly from the one obtained in the isospin basis (labelled by $(\mathrm{I})$ ).

Recently a thorough study of the $K^{-} p$ scattering length and its theoretical uncertainties within chiral 
Table 1. $\bar{K} N$ results of the Jülich model [14]. The value for the $K^{-} p$ scattering length obtained in the isospin-symmetric calculation is marked with (I). In case of kaonic hydrogen we present the strong-interaction energy shift $\Delta E$ and the width $\Gamma$ of the $1 s$ level. The result for the Jülich model is obtained from the $K^{-} p$ scattering length with the modified Deser-Trueman formula [41].

\begin{tabular}{|c|c|c|}
\hline & Jülich model [14] & Experiment \\
\hline \multicolumn{3}{|c|}{ Scattering lengths $[\mathrm{fm}]$} \\
\hline$a_{I=0}$ & $-1.21+i 1.18$ & \\
\hline$a_{I=1}$ & $1.01+i 0.73$ & \\
\hline$a_{K^{-} p}(\mathrm{I})$ & $-0.10+i 0.96$ & \\
\hline$a_{K^{-} p}$ & $-0.36+i 1.15$ & \\
\hline \multicolumn{3}{|c|}{ Kaonic hydrogen } \\
\hline \multirow[t]{2}{*}{$\Delta E$} & $217 \mathrm{eV}$ & $323 \pm 63 \pm 11 \mathrm{eV}[44,45]$ \\
\hline & & $193 \pm 37 \pm 6 \mathrm{eV}[42,43]$ \\
\hline \multirow[t]{2}{*}{$\Gamma$} & $849 \mathrm{eV}$ & $407 \pm 208 \pm 100 \mathrm{eV}[44,45]$ \\
\hline & & $249 \pm 111 \pm 39 \mathrm{eV}[42,43]$ \\
\hline \multicolumn{3}{|c|}{ Threshold ratios - eq. (2) } \\
\hline$\gamma$ & 2.30 & $2.36 \pm 0.04[48,49]$ \\
\hline$R_{c}$ & 0.65 & $0.664 \pm 0.011[48,49]$ \\
\hline$R_{n}$ & 0.22 & $0.189 \pm 0.015[48,49]$ \\
\hline \multicolumn{3}{|c|}{ Pole positions $[\mathrm{MeV}]$} \\
\hline$S_{01}$ & $1435.8+i 25.6$ & \\
\hline$S_{01}$ & $1334.3+i 62.3$ & \\
\hline
\end{tabular}

$S U(3)$ unitary approaches was presented [40]. The full calculation which included the WT contact interaction at leading chiral order, the direct and crossed Born terms as well as contact interactions from the Lagrangian of second chiral order yielded a scattering length of $a_{K^{-}}=$ $-1.05+i 0.75 \mathrm{fm}$. Obviously, the result obtained for the Jülich meson-exchange model differs from this value, but it is still within the $1 \sigma$ confidence region given in ref. [40].

Results for the strong-interaction energy shift $\Delta E$ and the width $\Gamma$ of the $1 s$ level of kaonic hydrogen, deduced from the $K^{-} p$ scattering length with the modified DeserTrueman formula [41], are also given in table 1. Interestingly, the prediction of the Jülich model for $\Delta E$ agrees well with the DEAR result $[42,43]$ while $\Gamma$ is roughly in line with the value found in the KEK experiment $[44,45]$. Note, however, that the experimental results for kaonic hydrogen were not available at the time when the Jülich $\bar{K} N$ model was constructed and, therefore, not included in the fitting procedure. Since the KEK and DEAR results are not compatible with each other it is important to resolve this discrepancy between the experimental results. Values of higher level of precision are expected to be reached by the ongoing SIDDHARTA experiment at LNF $[46,47]$.

Of interest are also the three measured threshold ratios $[48,49]$ of the $K^{-} p$ system, which are defined by

$$
\begin{aligned}
\gamma & =\frac{\Gamma\left(K^{-} p \rightarrow \pi^{+} \Sigma^{-}\right)}{\Gamma\left(K^{-} p \rightarrow \pi^{-} \Sigma^{+}\right)}, \\
R_{c} & =\frac{\Gamma\left(K^{-} p \rightarrow \text { charged particles }\right)}{\Gamma\left(K^{-} p \rightarrow \text { all }\right)}, \\
R_{n} & =\frac{\Gamma\left(K^{-} p \rightarrow \pi^{0} \Lambda\right)}{\Gamma\left(K^{-} p \rightarrow \text { all neutral states }\right)} .
\end{aligned}
$$

The corresponding predictions of the Jülich model are given in table 1. As one can see, the values are remarkably close to the experimental ones, specifically when one keeps in mind that these threshold ratios were not included in the fitting procedure when the model was constructed.

Re-calculating total cross sections in the particle basis yielded results that do not differ very much from those shown in ref. [14] (obtained in isospin basis) for momenta $p_{\text {lab }} \gtrsim 100 \mathrm{MeV} / c$ where data are available. In particular, the changes are small in comparison to the given experimental error bars. Thus, we do not present the corresponding results here.

Let us now come to the $\Lambda(1405)$. As already said above, also in the Jülich model this structure is generated dynamically. It is predicted "unambiguously" [14] to be a quasi-bound $\bar{K} N$ state. When searching in the region between the $\pi \Sigma$ and $\bar{K} N$ threshold we find two poles in the complex plane for the relevant $S_{01}$ partial wave. The values of the pole positions are listed in table 1 . In view of the extensive discussion of the two-pole structure of the $\Lambda(1405)$ over the last ten years or so $[40,50-55]$ it is certainly not surprising that the Jülich meson-exchange potential of the $\bar{K} N$ interaction generates two such poles as well. But back in 1989 when the model was constructed this was not an issue yet. Thus, no attempt was made to examine the pole structure of the amplitude in detail and, therefore, this feature remained undiscovered.

The pole structure predicted by the Jülich model turns out to be quantitatively very similar to the one of the full result of the chiral $S U(3)$ unitary approach by Borasoy et al. [40]. One pole, the $\bar{K} N$ "bound state", is located fairly close to the $\bar{K} N$ threshold and to the physical real axis while the other one is close to the $\pi \Sigma$ threshold and has a significantly larger imaginary part. It was emphasized in refs. $[40,54]$ that only the pole close to the physical axis remains relatively fixed while the other one depends sensitively on the dynamics included in the analysis, on the values chosen for inherent parameters, etc. Indeed, a glance at the published results for the poles predicted by different interactions $[50-53,56]$ reveals that there are significant variations in the values for the positions and widths. For example, the interactions proposed in refs. [50, $53]$ lead to two poles that are both very close to the physical region. In case of the WT models of refs. [51,52,56] the second pole shows a larger width, more in line with our results and those of refs. [40,54], though the two poles are still fairly close together. 

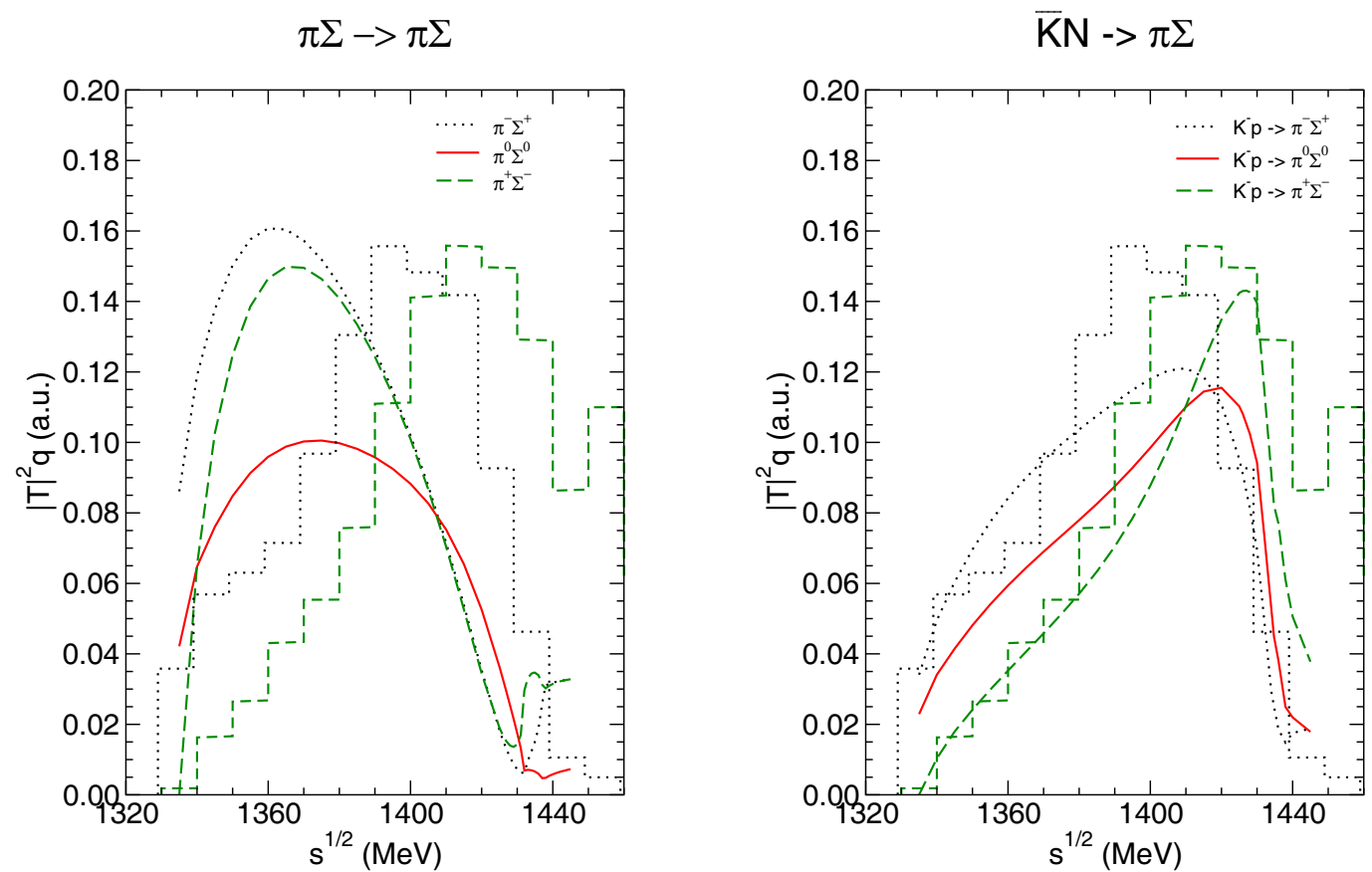

Fig. 3. $\pi \Sigma$ invariant-mass spectrum. On the left are results based on the $\pi \Sigma \rightarrow \pi \Sigma T$-matrix and on the right the ones for $K^{-} p \rightarrow \pi \Sigma$. The histograms are experimental results for the $\pi^{-} \Sigma^{+}$(dotted line) and $\pi^{+} \Sigma^{-}$(dashed line) channels taken from ref. [57].

In ref. [14] results for the quantity $\left|T_{\pi \Sigma}\right|^{2} \cdot q$ were presented, which is commonly associated and compared with the $\pi \Sigma$ mass distributions, i.e.

$$
\frac{\mathrm{d} \sigma}{\mathrm{d} m_{\pi \Sigma}} \propto\left|T_{\pi \Sigma}\right|^{2} q
$$

Here, $q$ is the center-of-mass momentum of the $\pi \Sigma$ system. Measurements of the $\pi \Sigma$ invariant-mass distribution for reactions like $\bar{K} N \rightarrow \pi \pi \pi \Sigma[57]$ or $\pi N \rightarrow$ $K \pi \Sigma[58]$ provide the main experimental evidence for the $\Lambda(1405)$-resonance. As we realize now, the results published in ref. [14] were not correct, because of an error in the phase-space factor in the computer code. Moreover, in this reference only the contribution from $I=0$ alone was considered.

In fig. 3 we present results for different charge channels $\left(\pi^{-} \Sigma^{+}, \pi^{0} \Sigma^{0}, \pi^{+} \Sigma^{-}\right)$in the final state and consider $\pi \Sigma \rightarrow \pi \Sigma$ as well as $\bar{K} N \rightarrow \pi \Sigma$ transitions, and compare them with the $\pi \Sigma$ mass distribution measured in the reaction $K^{-} p \rightarrow \pi \pi \pi \Sigma$ [57]. We display here curves for the individual $T$-matrices because we want to illustrate the differences between the various amplitudes. Note that the "true" amplitude to be inserted in eq. (3) will be a coherent sum of transition amplitudes from all allowed intermediate states to a specific final state, weighted with coefficients that reflect the details of the reaction mechanism [50].

As already observed by others in the past $[52,59]$, there is a remarkable difference between the invariantmass spectrum due to the $\pi \Sigma \rightarrow \pi \Sigma$ amplitude (left-hand side of fig. 3) and the one due to the $\bar{K} N \rightarrow \pi \Sigma$ amplitude (right-hand side of fig. 3). This is due to the fact that the two poles in the $I=0 S$-wave amplitude have different widths and couple also differently to the $\bar{K} N$ and $\pi \Sigma$ channels [52]. In case of the Jülich potential the former shows little resemblance with the $\pi \Sigma$ invariant-mass spectrum given in ref. [57] while the results based on the $\bar{K} N \rightarrow \pi \Sigma$ amplitude are roughly in line with the empirical information.

Note that there are also significant differences in the invariant-mass distributions due to the $K^{-} p \rightarrow \pi \Sigma$ amplitudes for the different possible charge states of $\pi \Sigma$. Specifically, the peak positions for $\pi^{-} \Sigma^{+}$and $\pi^{+} \Sigma^{-}$differ by almost $30 \mathrm{MeV}$. Interestingly, the experimental invariantmass distributions for the two charge states, cf. the dotted and dashed histograms in fig. 3, respectively, seem to show a similar separation of their peak and both agree roughly with the corresponding predictions of the Jülich $\bar{K} N$ potential. We should mention, however, that the experimental $\pi^{+} \Sigma^{-}$mass spectrum is afflicted by fairly large background contributions [57].

These differences in the invariant-mass distributions are caused primarily by the interference between the $I=0$ and $I=1$ amplitudes [60] when evaluating the observables in the particle basis. They are not due to isospin-breaking effects. Indeed, we found that an isospin-symmetric calculation based on averaged masses yields very similar results. Note that then the $\pi^{-} \Sigma^{+} \rightarrow \pi^{-} \Sigma^{+}$and $\pi^{+} \Sigma^{-} \rightarrow \pi^{+} \Sigma^{-}$ results would coincide.

In this context let us mention that there have been some recent experimental attempts to achieve a better determination of the $\pi \Sigma$ invariant-mass spectrum in the region of the $\Lambda(1405)[61,62]$. The $\pi^{0} \Sigma^{0}$ distribution measured in the reaction $K^{-} p \rightarrow \pi^{0} \pi^{0} \Sigma^{0}$ by the Crystal Ball 
Table 2. $D N$ results of the meson-exchange model, the $S U(4)$ WT model of ref. [28] and the $S U(8)$ WT model of ref. [30]. The pole positions of the $S U(4)$ WT model were published in [30].

\begin{tabular}{|c|c|c|c|}
\hline & Meson-exchange model & $S U(4) D N$ model $[28]$ & $S U(8) D N$ model $[30]$ \\
\hline \multicolumn{3}{|c|}{ Scattering lengths $[\mathrm{fm}]$} \\
\hline$a_{I=0}$ & $-0.41+i 0.04$ & $-0.57+i 0.001$ & $0.004+i 0.002$ \\
$a_{I=1}$ & $-2.07+i 0.57$ & $-1.47+i 0.65$ & $0.33+i 0.05$ \\
\hline \multicolumn{3}{|c|}{ Pole positions [MeV] } \\
\hline$S_{01}$ & $2593.9+i 2.88$ & $2595.4+i 1.0$ & $2595.4+i 0.3$ \\
$S_{01}$ & $2603.2+i 63.1$ & $2625.4+i 51.5$ & $2610.0+i 35.5$ \\
$S_{01}$ & & $2799.5+i 0.0$ & $2821.5+i 0.5$ \\
$S_{01}$ & $2797.3+i 5.86$ & $2661.2+i 18.2$ & $2871.2+i 45.6$ \\
$S_{11}$ & & $2694.7+i 76.5$ & $2553.6+i 0.34$ \\
$S_{11}$ & & & $2612.2+i 89.5$ \\
$S_{11}$ & & & $2837.1+i 40.0$ \\
$S_{11}$ & & & $2868.0+i 19.3$ \\
$S_{11}$ & & & \\
$P_{01}$ & & & \\
\hline
\end{tabular}

Collaboration [61] has been analyzed in ref. [63] within the chiral unitary approach and, together with corresponding results for the reaction $\pi^{-} p \rightarrow K^{0} \pi \Sigma$ [64], is considered as evidence of the two-pole nature of the $\Lambda(1405)$ [63]. The interest in the lineshape of the $\Lambda(1405)$ has been again revived with the $\Lambda(1405)$ photoproduction experiment on a proton target at CLAS@JLAB. This experiment is providing the first results on the $1(1405)$ photoproduction analyzing all three $\pi \Sigma$ decay modes. Preliminary results show differences in the $\Lambda(1405)$ lineshapes as well as different $\Lambda(1405)$ differential cross sections for each $\pi \Sigma$ decay mode $[65,66]$.

\section{Results for DN}

As already stated above, the $S U(4)$ extension of the Jülich $\bar{K} N$ model to the $D N$ interaction generates narrow states in the $S_{01}$ and $S_{11}$ partial waves which we identify with the experimentally observed $\Lambda_{c}(2595)$ and $\Sigma_{c}(2800)$ resonances, respectively. Not surprisingly, we find an additional pole in the $S_{01}$ partial wave, located close to the other one. Similar to the corresponding state in the $\bar{K} N$ sector, the second pole has a much larger width, cf. table 2. Interestingly, our model even generates a further state, namely in the $P_{01}$ partial wave, which, after finetuning (cf. sect. 2), lies at $2804 \mathrm{MeV}$, i.e. just below the $D N$ threshold. We are tempted to identify this state with the $\Lambda_{c}(2765)$-resonance, whose quantum numbers are not yet established [25]. Though we do not reproduce the resonance energy quantitatively, we believe that further refinements in the $D N$ model, specifically the inclusion of the $\pi \pi \Lambda_{c}$ channel in terms of an effective $\sigma \Lambda_{c}$ channel, can provide sufficient additional attraction for obtaining also quantitative agreement. The mechanism could be the same as in the case of the Roper $\left(N^{*}(1440)\right)$ reso- nance, which is generated dynamically in the Jülich $\pi N$ model $[67,68]$. Here the required strong attraction is produced via the coupling of the $\pi N P$-wave (where the Roper occurs) to the $S$-wave in the $\sigma N$ system, facilitated by the different parities of the $\pi$ and $\sigma$ mesons. Besides shifting the resonance position, the coupling to an effective $\sigma \Lambda_{c}$ would certainly also increase the width significantly, as is required for a reproduction of the experimental information [25].

For comparison we include here some predictions of the $D N$ interactions presented in refs. $[28,30]$ which are based on the leading-order WT contact term. One of them [28] is derived in an $S U(4)$ framework comparable to our meson-exchange model, while the other one is based on an extended $S U(8)$ spin-flavor scheme [30]. Both these $D N$ interactions have also been adjusted to reproduce the $\Lambda_{c}(2595)$-resonance. However, compared to the Jülich meson-exchange model, the total number of poles and their energies are different, cf. table 2 . Three $S_{01}$ and two $S_{11}$ states appear up to $2900 \mathrm{MeV}$ in the $S U(4) D N$ WT model, as reported in ref. [30]. Among them, there is a $S_{11}$ state at $2694 \mathrm{MeV}$ with a width of $\Gamma=153 \mathrm{MeV}$ that strongly couples to the $D N$ channel. Thus, it is observed as a structure in the real axis close to the $D N$ threshold with similar effects as the $S_{11}$-resonance at $2797 \mathrm{MeV}$ of our model. The $S U(8) D N$ WT model [30] predicts even more states in this energy region, as indicated in table 2. But it also yields a $\Lambda_{c}(2595)$-resonance with a significantly smaller width than the other two interaction models. This has consequences for the $\pi \Sigma_{c}$ invariant-mass spectrum, to be discussed below.

Some results for the $D N$ scattering are presented in figs. 4 and 5. Specifically, we show $D N$ cross sections for $I=0$ and $I=1$ based on the parameter set that reproduces the positions of the $\Lambda_{c}(2595)$ and $\Sigma_{c}(2800)$ of the Particle Data Group (solid line) together with re- 

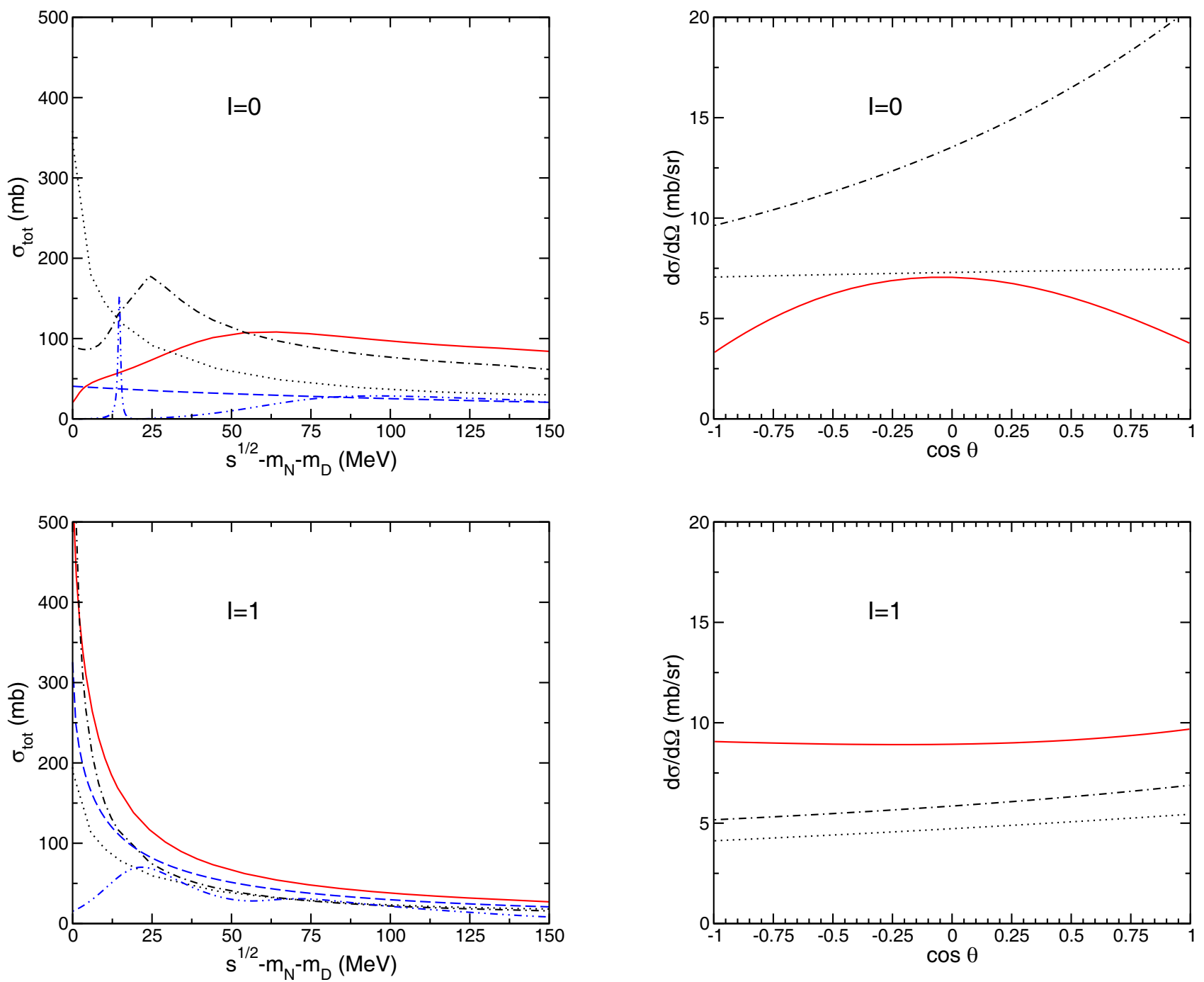

Fig. 4. $D N$ cross sections for the isospin $I=0$ (top) and $I=1$ (bottom) channels as a function of $\epsilon=\sqrt{s}-m_{N}-m_{D}$. The solid lines are the results of our meson-exchange model. The dash-dotted lines are based on parameters taken directly from earlier $\bar{K} N$ and $K N$ potentials $[15,16,38]$, cf. text, while the dashed lines are predictions of the $S U(4)$ WT model of, ref. [28] and the dash-double-dotted lines are predictions of the $S U(8)$ WT model of ref. [30]. The dotted lines are the corresponding results for $\bar{K} N$ of the Jülich meson-exchange model [14].

sults that follow directly from the $\bar{K} N$ and $K N$ studies of refs. $[15,16,38]$ by assuming $S U(4)$ symmetry (dashdotted line). The $D N$ cross sections of the $S U(4) \mathrm{WT}$ model of ref. [28] (dashed line) and of the $S U(8)$ WT model of ref. [30] (dash-double-dotted line) are also displayed. Finally, we include the $\bar{K} N$ cross sections of the original Jülich model [14] for reference (dotted line).

Obviously, the $D N$ cross sections show a significant momentum dependence. Furthermore, they are substantially larger than those we obtain for $\bar{D} N$ [4]. In particular, the cross section in the isospin $I=1$ channel amounts to almost $600 \mathrm{mb}$ at threshold. This is not too surprising in

Fig. 5. Differential cross sections for $D N$ and $\bar{K} N$ for the isospin $I=0$ (top) and $I=1$ (bottom) channels at $\epsilon=$ $25 \mathrm{MeV}$. The solid lines are the results of our meson-exchange model. The dash-dotted lines are based on parameters taken directly from earlier $\bar{K} N$ and $K N$ potentials [15,16,38], cf. text, while the dotted lines are the corresponding results for $\bar{K} N$ of the Jülich meson-exchange model [14].

view of the near-by $S_{11}$ quasi-bound state. The structure of the cross section in the isospin $I=0$ channel is strongly influenced by the $P_{01}$ partial wave where our model produces a near-threshold quasi-bound state or, in case of the parameter set directly fixed by $S U(4)$ constraints, a resonance state around $25 \mathrm{MeV}$ above threshold. The $D N$ cross section of the $S U(4)$ WT approach of ref. [28] shows a similar behaviour as the one of the Jülich model for the $I=1$ channel. As already discussed above, this model generates likewise poles in the $S_{11}$ partial wave, though not as close to the $D N$ threshold as the Jülich model. In case of the $I=0$ channel there are pronounced differences. But this is not surprising because the $S U(4)$ WT model yields only $S$-wave contributions while the results of the Jülich 
model are dominated by the $P$-wave. Overall larger differences are seen in comparison to the results for the $S U(8)$ WT model. Specifically, for $I=0$ it predicts cross sections close to zero in the near-threshold region and one sees a clear resonance signal from the narrow state generated at around $2821 \mathrm{MeV}$, cf. table 2 .

Please note that the $S U(8)$ WT model of [30] is not just a straight extension of the $S U(4)$ WT model [28]. For example, the regularization procedures are different: in the $S U(4)$ WT model a subtraction at a certain scale is implemented while for the $S U(8)$ WT model the subtraction is performed slightly below the lowest threshold. Furthermore, the $S U(4)$ symmetry is broken in different ways. In the $S U(4)$ WT model the symmetry is broken primarily by the used physical masses and by a fixed reduction factor in the (non-diagonal) transitions where a heavy (charmed) meson can be exchanged. In the $S U(8)$ WT model the symmetry is broken by using physical masses but also due to the different weak decay constants used, cf. the discussion in [30]. In the Jülich model the $D^{*} N$ channel is already explicitly included as required by HQS. However, unlike in the $S U(8)$ WT model the direct interaction in the $D^{*} N$ system is ignored. Nonetheless, one should not expect that an extension of the Jülich model with full implementation of HQS, which is certainly an interesting project for the future, will lead to results similar to the $S U(8)$ WT model. Realizing that there is a significant cancellation between the $\pi$ and $\rho$ exchanges (that strongly reduces the net effect of their important tensor components) it has been the strategy of the Bonn and Jülich groups to include always both contributions. This philosophy was also followed in the strangeness sector [14-16] and is followed now in the charm case too. As a consequence, the $D N \rightarrow D^{*} N$ transition potential due to the combined $\pi$ and $\rho$ exchange is fairly weak - and the same would happen for the corresponding (direct) $D^{*} N \rightarrow D^{*} N$ interaction. In the $S U(8)$ WT model of [30] only the WT term is included, corresponding to vector-meson $(\rho)$ exchange, for $D^{*} N \rightarrow D^{*} N$ as well as for $D N \rightarrow D^{*} N$ suggesting that those interactions should be significantly stronger than what we would get in the meson-exchange picture.

Let us now come to the scattering lengths. The discussed quasi-bound state in the $S_{11}$ channel of the mesonexchange model is also reflected in the pertinent results, cf. table 2 , namely by the rather large value of the real part of $a_{I=1}$. The same situation is observed in the $S U(4) \mathrm{WT}$ approach of [28]. In fact, the $S$-wave scattering lengths predicted by our model and by the WT approach turn out to be very similar qualitatively for the $I=1$ as well as for the $I=0$ channel, as can be seen in table 2 . This cannot be said about the $S U(8)$ WT model of [30] which predicts radically different scattering lengths in both isospin channels. (Note that there is a typo in the real part of the $I=0$ scattering length in ref. [69], where the results were first published.) For completeness, let us mention here that the scattering lengths of the $D N$ interaction of Hofmann and Lutz [27], reported in ref. [29], amount to about $-0.4 \mathrm{fm}$ for both isospin channels. In agreement with that work we find that the imaginary part in the Jülich model is negli- gibly small for $I=0$. However, the imaginary part in the $I=1$ channel for our $D N$ model is not negligible, contrary to ref. [29]. This is due to the fact that in ref. [29] there is no quasi-bound state close to $D N$ threshold, but lies $180 \mathrm{MeV}$ below.

Angular distributions for the reaction $D N \rightarrow D N$ are shown in fig. 5. Obviously, in the $I=0$ case there is a strong anisotropy already at fairly low momenta. It is due to significant contributions in the $P_{01}$ partial wave in this momentum region induced by the near-threshold quasibound state or resonance, respectively, produced by our model, as discussed above. For higher momenta, the differential cross section becomes forward peaked, similar to the predictions of our model for the $\bar{D} N$ system [4].

Predictions for $D N$ scattering observables in the particle basis $\left(D^{0} n \rightarrow D^{0} n, D^{0} p \rightarrow D^{0} p, D^{0} p \rightarrow D^{+} n\right)$ can be found in ref. [5].

\section{Discussion of the $\Lambda_{c}(2595)$}

The excited charmed baryon $\Lambda_{c}(2595)$ was first observed by the CLEO Collaboration [21] and its existence was later confirmed in experiments by the E687 [22] and ARGUS [23] Collaborations. In all these experiments the resonance appears as a pronounced peak in the invariant-mass distribution of the $\pi^{+} \pi^{-} \Lambda_{c}^{+}$channel.

It is now generally accepted that the $\Lambda_{c}(2595)$ is the charmed counterpart of the $1(1405)$ [25]. Therefore, it is natural that interaction models of the $\bar{K} N$ system in which the $\Lambda(1405)$ appears as a dynamically generated state, as is the case in chiral unitary approaches as well as in the traditional meson-exchange picture, likewise generate the $\Lambda_{c}(2595)$ dynamically, provided that $S U(4)$ symmetry is assumed when extending the interactions from the strangeness to the charm sector. In this context it is important to realize that there are also drastic differences between the strangeness and the charm case. In particular, the $\Lambda(1405)$ is located fairly close to the $\bar{K} N$ threshold, which is around $1430 \mathrm{MeV}$, while the $\Lambda_{c}(2595)$ coincides practically with the $\pi \Sigma_{c}$ threshold, which is at around $2593 \mathrm{MeV}$. Furthermore, the $\pi \Sigma$ and $\bar{K} N$ thresholds are roughly $100 \mathrm{MeV}$ apart, while there are more than $200 \mathrm{MeV}$ between the $\pi \Sigma_{c}$ and $D N$ thresholds. Finally, the $\pi \pi \Lambda_{c}$ channel — where the $\Lambda_{c}(2595)$ is experimentally observed-opens $35 \mathrm{MeV}$ below the resonance, while the corresponding $\pi \pi \Lambda$ channel is barely open at the location of the $\Lambda(1405)$. Note that the $\pi \pi$ channel must be in the $I^{G}\left(J^{P}\right)=0^{+}\left(0^{+}\right)(" \sigma ")$ state when $\pi \pi \Lambda_{c}$ couples to the $\Lambda_{c}(2595)$. And, due to parity conservation, it is the $P_{01}$ partial wave of the $\pi \pi \Lambda_{c}(\pi \pi \Lambda)$ system which couples to the $S_{01} D N(\bar{K} N)$ channel.

In view of the mentioned kinematical differences, it is certainly not surprising that the models do not really predict the $\Lambda_{c}(2595)$ at exactly the position where it was found in the experiment. A fine-tuning of inherent parameters such as subtraction constants or coupling constants is required to shift the resonance to the observed energy. Specifically, in case of the Jülich $D N$ model the coupling constants of the scalar mesons were adjusted in such a way 

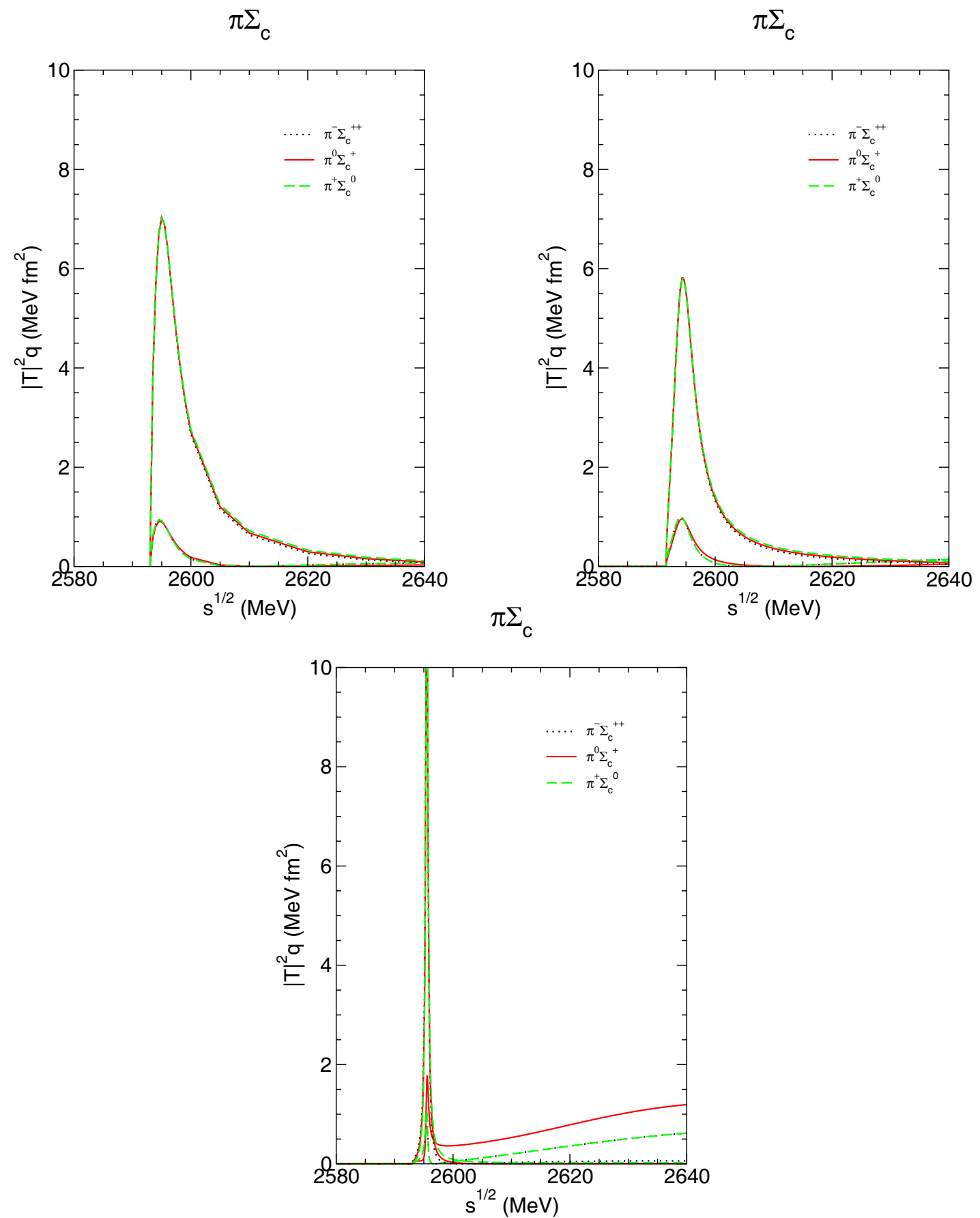

Fig. 6. $\pi \Sigma_{c}$ invariant-mass spectrum in an isospin-symmetric calculation. In the top-left panel are results based on our mesonexchange model. Those in the top-right panel are for the $S U(4)$ WT model of ref. [28] while those at the bottom are for the $S U$ (8) WT model of ref. [30]. The lower curves are based on the $\pi \Sigma_{c} \rightarrow \pi \Sigma_{c} T$-matrix while the upper ones correspond to $D^{0} p \rightarrow \pi \Sigma_{c}$.

that the $\pi \Sigma_{c} S$-wave phase shift in the $I=0$ channel goes through $90^{\circ}$ at $2595 \mathrm{MeV}$, i.e. at the nominal $\Lambda_{c}(2595)$ mass as listed in the PDG [25]. It should be said, however, that for an investigation of the $D N$ interaction, as performed in ref. [5], the precise position of the $\Lambda_{c}(2595)$ resonance does not play a role.

The experimental papers report uniformly that the $\Lambda_{c}(2595)$ decays dominantly into the $\pi^{+} \Sigma_{c}^{0}$ and $\pi^{-} \Sigma_{c}^{++}$ channels [22-24]. In the latter reference one can even read that $\Lambda_{c}(2595)$ decays almost $100 \%$ to $\pi \Sigma_{c}$. At first sight this seems not unreasonable. Considering the reported mass difference $M\left(\Lambda_{c}(2595)\right)-M\left(\Lambda_{c}\right)$ of

$$
\begin{aligned}
& 307.5 \pm 0.5 \pm 1.2 \mathrm{MeV}(\text { CLEO ‘95), } \\
& 309.7 \pm 0.9 \pm 0.4 \mathrm{MeV}(\text { E687'96), } \\
& 309.2 \pm 0.7 \pm 0.3 \mathrm{MeV} \text { (ARGUS '97), }
\end{aligned}
$$


respectively, and the corresponding threshold values for the $\pi \Sigma_{c}$ channels

$$
\begin{gathered}
M\left(\pi^{-}\right)+M\left(\Sigma_{c}^{++}\right)-M\left(\Lambda_{c}\right)=307.13 \pm 0.18 \mathrm{MeV}, \\
M\left(\pi^{0}\right)+M\left(\Sigma_{c}^{+}\right)-M\left(\Lambda_{c}\right)=301.42 \pm 0.4 \mathrm{MeV}, \\
M\left(\pi^{+}\right)+M\left(\Sigma_{c}^{0}\right)-M\left(\Lambda_{c}\right)=306.87 \pm 0.18 \mathrm{MeV}
\end{gathered}
$$

where we use the latest values from the PDG [25], there is some phase space for the $\Lambda_{c}(2595) \rightarrow \pi \Sigma_{c}$ decay.

However, the new CLEO measurement with improved statistics and with better momentum resolution [24] suggests a mass difference of only

$$
305.3 \pm 0.4 \pm 0.6 \mathrm{MeV} \text { (CLEO ‘99) }
$$

A very similar mass difference $(305.6 \pm 0.3 \mathrm{MeV})$ was obtained in an independent analysis of the new CLEO data by Blechman et al. [70]. Such a value reduces the phase space for the decay of the $\Lambda_{c}(2595)$ into the $\pi^{+} \Sigma_{c}^{0}$ and $\pi^{-} \Sigma_{c}^{++}$channels significantly. Indeed the decay is only possible due to the finite widths of the involved particles. But, since the widths are only in the order of $2 \mathrm{MeV}\left(\Sigma_{c}^{++}\right.$, $\left.\Sigma_{c}^{0}\right)$ to $4 \mathrm{MeV} \Lambda_{c}(2595)$ [25] and the detector resolution is $1.28 \mathrm{MeV}$ [24], it is still surprising that the $\Lambda_{c}(2595)$ should decay domaninatly into those two channels as suggested by the experiment. We will come back to this issue at the end of this section.

In the following we present predictions of the Jülich $D N$ model and of the $S U(4)$ and $S U(8)$ WT models of refs. $[28,30]$ for the invariant-mass distributions, i.e. for the quantity $|T|^{2} \cdot q$, in the relevant $\pi \Sigma_{c}$ channels. This allows us to explore whether there are any quantitative or even qualitative differences in the predictions of those models. Furthermore, we can illustrate the subtle effects of the slightly different thresholds of the $\pi^{+} \Sigma_{c}^{0}, \pi^{0} \Sigma_{c}^{+}$, and $\pi^{-} \Sigma_{c}^{++}$channels on the various invariant-mass distributions due to the presense of a near-by pole. But first, let us show results for the isospin-symmetric calculation (based on averaged masses), cf. fig. 6. The upper curves correspond to the $D N \rightarrow \pi \Sigma_{c} T$-matrix while the lower ones are for $\pi \Sigma_{c} \rightarrow \pi \Sigma_{c}$. In this figure the relative normalization of the $\pi \Sigma_{c}$ to the $D N \rightarrow \pi \Sigma_{c}$ channel is kept as predicted by the models but all $T$-matrices are multiplied with the mass factor $M_{\pi} M_{\Sigma_{c}} /\left(M_{\pi}+M_{\Sigma_{c}}\right)$ in order to obtain convenient units for plotting.

The results shown in fig. 6 make clear that in case of our meson-exchange model the amplitudes of the $D N-\pi \Sigma_{c}$ systems are completely dominated by the $I=0$ contribution in the region of the $\Lambda_{c}(2595)$. As a consequence, the predictions for all charge states practically coincide. As mentioned above, this is not the case for $\bar{K} N-\pi \Sigma$ where the interference between the $I=0$ and $I=1$ amplitudes is significant. For example, there the invariant-mass distribution for $\pi^{ \pm} \Sigma^{\mp} \rightarrow \pi^{ \pm} \Sigma^{\mp}$ differs by roughly a factor of two from that of $\pi^{0} \Sigma^{0} \rightarrow \pi^{0} \Sigma^{0}$, cf. fig. 3. Furthermore, it can be seen from fig. 6 that the shape of the invariantmass distribution obtained from the $\pi \Sigma_{c}$ and $D N \rightarrow \pi \Sigma_{c}$ $T$-matrices are fairly similar. In fact, a comparison of the results shown in the left and right panels of fig. 6 reveals that there is even hardly any difference between the results of the meson-exchange model and the $S U(4) \mathrm{WT}$ interaction. Even the absolute magnitudes are rather similar. This might be surprising but is certainly a reflection of the specific situation with the $\Lambda_{c}(2595)$ being located very close to the $\pi \Sigma_{c}$ threshold. In such cases one expects to see features that are practically model independent [71-73].

On the other hand, the results for the $S U(8) \mathrm{WT}$ model differ drastically. This is due to the fact that the $\Lambda_{c}(2595)$ generated by this model is much narrower, cf. table 2 , and the near-by second state in the $I=0$ sector is also narrower than in the other two models considered. Moreover, there is even a near-by state in the $I=1$ channel (at $2612 \mathrm{MeV}$ ) so that the $I=1$ amplitude is sizeable - in contradistinction to what happens for the Jülich and the $S U(4)$ WT interactions. In this context let us mention that the $D N \rightarrow \pi \Sigma_{c}$ results of the Jülich model are calculated from the half-off-shell transition $T$-matrix assuming the $D N$ momentum to be zero, while for the WT results $[28,30]$ the on-shell amplitude is used. In the latter case the corresponding $D N$ momentum is purely imaginary.

Results based on the physical masses are presented in fig. 7. Here the invariant-mass distributions are shown in arbitrary units and normalized in such a way that one can easily compare the results based on the $\pi \Sigma_{c} \rightarrow \pi \Sigma_{c}$ (left panel) and $D N \rightarrow \pi \Sigma_{c}$ (right panel) $T$-matrices. But we keep the relative normalization between the different charge channels as predicted by the model. Obviously, there are drastic effects due to the different thresholds. The threshold of the $\pi^{0} \Sigma_{c}^{+}$channel is about $6 \mathrm{MeV}$ lower than those of the other two charge channels and, as a consequence, the predicted invariant-mass distribution is about twice as large. Moreover, there is a clear cusp in $\pi^{0} \Sigma_{c}^{+}$at the opening of the $\pi^{+} \Sigma_{c}^{0}$ channel. The threshold of $\pi^{-} \Sigma_{c}^{++}$is just about $0.3 \mathrm{MeV}$ above the one for $\pi^{+} \Sigma_{c}^{0}$. It produces a noticeable kink in the $\pi^{0} \Sigma_{c}^{+}$invariant-mass distribution. On the other hand, and as already anticipated from the comparison of the isospin-symmetry calculation above, there are only rather subtle differences between the lines shapes predicted from the $\pi \Sigma_{c} \rightarrow \pi \Sigma_{c}$ and from the corresponding $D N \rightarrow \pi \Sigma_{c}$ amplitudes. The only more qualitative difference consists in the stronger falloff with increasing invariant mass exhibited by the results based on the $\pi \Sigma_{c} \rightarrow \pi \Sigma_{c} T$-matrix.

Finally, let us come to the experimental $\pi^{+} \pi^{-} \Lambda_{c}^{+}$mass distribution where the signal for the $\Lambda(2595)$ was found. The corresponding data $[23,24]$ are also displayed in fig. 7. The results for the $D^{0} p \rightarrow \pi^{0} \Sigma_{c}^{+}$as well as for the $\pi^{0} \Sigma_{c}^{+} \rightarrow \pi^{0} \Sigma_{c}^{+}$channels resemble indeed very much the measured signal and one can imagine that smearing out our results by the width of the $\Sigma_{c}^{+}$, which is roughly $4 \mathrm{MeV}[24,25]$, would yield a fairly good fit to the data. However, experimentally it was found that the $\Lambda_{c}(2595)$ decays predominantly into the $\pi^{+} \Sigma_{c}^{0}$ and $\pi^{-} \Sigma_{c}^{++}$channels with a branching fraction in the range of $66 \%$ [23] to close to $100 \%$ [24]. Smearing out the corresponding results with the significantly smaller and better known widths of the $\Sigma_{c}^{0}$ and $\Sigma_{c}^{++}$, of just $2 \mathrm{MeV}$ [25], would still leave many of the events found below the nominal $\pi^{+} \Sigma_{c}^{0}$ and $\pi^{-} \Sigma_{c}^{++}$threshold unexplained, especially for the CLEO 

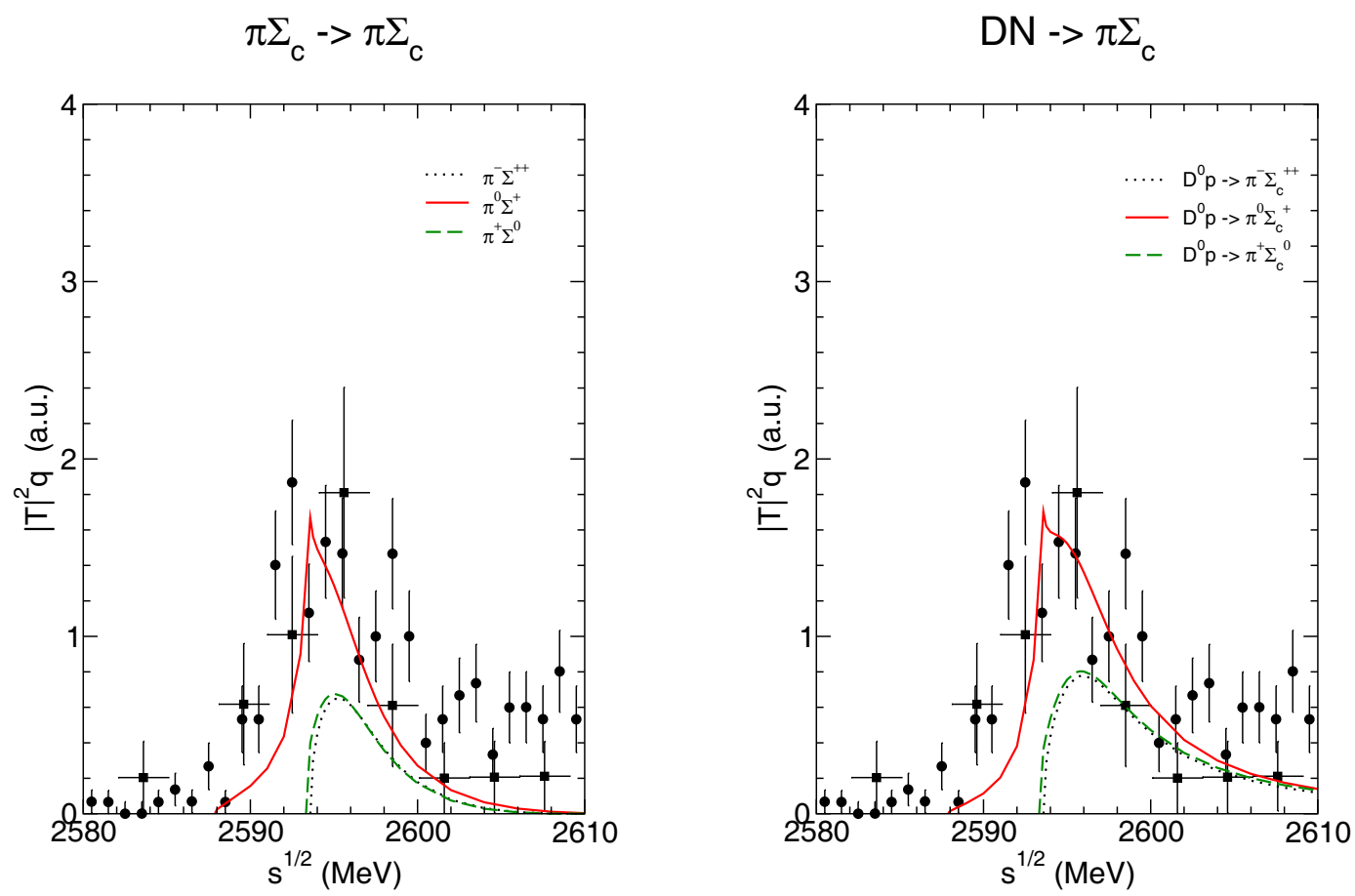

Fig. 7. $\pi \Sigma_{c}$ invariant-mass spectrum predicted by our $D N$ meson-exchange model. On the left are results based on the $\pi \Sigma_{c} \rightarrow \pi \Sigma_{c} T$-matrix and on the right the ones for $D^{0} p \rightarrow \pi \Sigma_{c}$. For illustration purposes we show also data for the $\pi^{+} \pi^{-} \Lambda_{c}^{+}$ invariant-mass distribution taken from [23] (squares) and [24] (circles).

experiment [24]. Thus, it seems to us that the position of the $\Lambda_{c}(2595)$-resonance being so close to or even below the nominal $\pi^{+} \Sigma_{c}^{0} / \pi^{-} \Sigma_{c}^{++}$thresholds and the found large branching ratios into those channels are difficult to reconcile.

Independently of that, we would like to say also a word of caution concerning our own results. In view of the fact that the signal for the $\Lambda_{c}(2595)$-resonance is seen in the $\pi^{+} \pi^{-} \Lambda_{c}^{+}$channel, any more rigorous model analysis would definitely require the explicit inclusion of this channel. In principle, the presence of the $\pi \pi \Lambda_{c}^{+}$channel could be simulated within our model by adding a phenomenological $\sigma \Lambda_{c}^{+}$ channel, analogous to the treatment of the $\pi \pi N$ channel in our $\pi N$ model $[67,68]$. But then many new parameters would have to be introduced that can no longer be fixed by $S U(4)$ arguments in a reasonable way.

In any case, first it would be important to confirm the new CLEO data by independent measurements of the $\pi \pi \Lambda_{c}^{+}$and $\pi \Sigma_{c}$ mass spectra in the region of the $\Lambda_{c}(2595)$. Specifically, it would be essential to establish unambigously that there is a large decay rate of that resonance into the $\pi \Sigma_{c}$ channels. A precise determination of the pole position of the $\Lambda_{c}(2595)$ could then be done along model-independent approaches such as the ones suggested in refs. [70,71]. Besides being much better suited for performing a fit to data and for deducing uncertainties, these approaches allow one to incorporate also finite widths effects appropriately which is very difficult to achieve in models like the ones discussed in the present paper. In view of the experimental situation discussed above such finite widths effects might play a crucial role.
Note that the predictions of the $S U(4)$ WT model for the quantities $\left|T_{\pi \Sigma_{c} \rightarrow \pi \Sigma_{c}}\right|^{2} \cdot q$ and $\left|T_{D^{0} p \rightarrow \pi \Sigma_{c}}\right|^{2} \cdot q$ are very similar to the ones of the meson-exchange model and, therefore, we do not show the corresponding curves here. On the other hand, those for the $S U(8)$ WT interaction are characterized by the very narrow width of the $\Lambda_{c}(2595)$ predicted by this model and, thus, do not resemble at all the measured $\pi \Sigma_{c}$ invariant-mass spectrum. We refrain from showing those results here.

In order to understand the differences in the mass spectra for the strangeness and charm sectors it is instructive to take a look at the phase shifts of the $\pi \Sigma$ and $\pi \Sigma_{c}$ channels in the $S_{01}$ partial wave where the poles corresponding to the $\Lambda(1405)$ and $\Lambda_{c}(2595)$ are located. Corresponding results are presented in fig. 8. The standard relation of the (partial-wave-projected) $T$-matrix to the phase shift is $T(q)=-\exp (i \delta(q)) \sin (\delta(q)) / q$. The quantity $\sin ^{2}(\delta(q))$ has its maximum where $\delta(q)$ passes through $90^{\circ}$. The maximum of the corresponding invariant-mass distribution, $|T|^{2} \cdot q=\sin ^{2}(\delta(q)) / q$, will occur at somewhat smaller invariant masses, due to the additional $1 / q$ factor and depending on the slope with which the phase shift passes through $90^{\circ}$. The invariant-mass distribution will be zero where the phase shift passes through $180^{\circ}$. These are indeed the features of the $\pi \Sigma \rightarrow \pi \Sigma$ invariant-mass spectrum, shown in fig. 3 on the left side. On the other hand, the $\bar{K} N \rightarrow \pi \Sigma$ invariant-mass spectrum has its maximum close to the position of the pole. This corresponds to the region where the $\pi \Sigma$ phase shift exhibits the strongest variation with energy, which is around 1420 to $1430 \mathrm{MeV}$, cf. fig. 8 (left). 

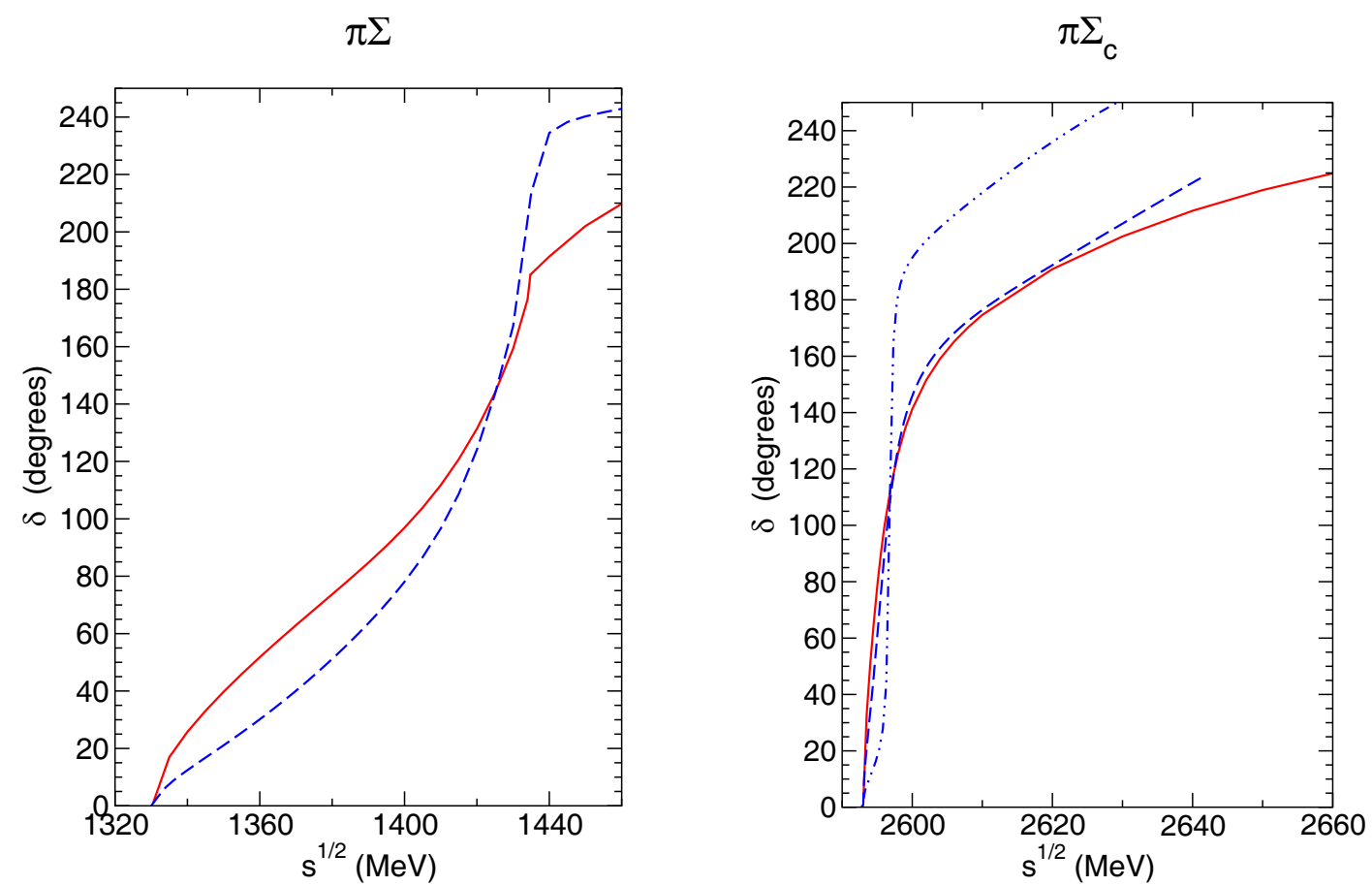

Fig. 8. $S_{01} \pi \Sigma$ and $\pi \Sigma_{c}$ phase shifts. Solid lines represent results for the Jülich $\bar{K} N$ and $D N$ models while the dashed lines are those for the corresponding WT interactions [28,56]. The dash-double-dotted line is for the $S U(8)$ WT interaction of [30].

In this context we would like to draw attention to the fact that the behavior of the $\pi \Sigma S_{01}$ phase shift and the pertinent invariant-mass distribution is very similar to the one of the $\pi \pi \delta_{00}$ partial wave. In the latter case the phase shift shows a broad shoulder at lower energies, passing slowly through $90^{\circ}$, a behavior usually associated with the $\sigma$-meson (or $f_{0}(600)$ ), while finally rising quicky through $180^{\circ}$ around $1 \mathrm{GeV}$ at the location of the $f_{0}(980)$ resonance. The corresponding mass spectrum consists in a broad bump on which the $f_{0}(980)$ appears as a dip structure, cf. ref. [74]. Also in the case of the $\bar{K} N-\pi \Sigma$ system the pole at around $1436 \mathrm{MeV}$ with the smaller width, which one might associate with the $\Lambda(1405)$, produces a peak in the $\bar{K} N \rightarrow \pi \Sigma$ invariant-mass spectrum but a dip in the one computed from the $\pi \Sigma \rightarrow \pi \Sigma T$-matrix.

The behavior of the corresponding phase shift for the $\pi \Sigma_{c}$ system is very much different, cf. fig. 8 (right). Here the strongest variation with energy occurs already very close to the threshold and in the same region the phase also goes through $90^{\circ}$.

The phase shift predicted by the $S U(4)$ WT model $[28$, $56]$ is shown by the dashed line in fig. 8. Results for $\pi \Sigma_{c}$ for the $S U(8)$ WT model [30] (dash-double-dotted line) are included too. Obviously, for the $D N-\pi \Sigma_{c}$ system the $S U(4)$ WT result is very similar to the prediction of the meson-exchange model. Thus, it is not surprising that also the corresponding invariant-mass distributions, presented in fig. 7 , are very similar to those of the Jülich potential. For $\bar{K} N-\pi \Sigma$ there are noticeable quantitative differences. In particular, the slope of the phase shift is significantly larger where it passes through $90^{\circ}$, reflecting the fact that the two poles of the WT model [56] are much closer to each other [52] than in case of the Jülich model, and, as a consequence, the maxima of the invariant-mass spectra based on the $\bar{K} N \rightarrow \pi \Sigma$ and $\pi \Sigma \rightarrow \pi \Sigma$ amplitudes are also closer to each other [52]. The $\pi \Sigma_{c}$ phase shift for the $S U(8)$ WT model clearly reflects the much narrower width of the $\Lambda_{c}(2595)$ (and of the close-by $S_{01} 2610 \mathrm{MeV}$ resonance) predicted by this interaction.

\section{Summary}

In this paper we presented a model for the interaction in the coupled systems $D N, \pi \Lambda_{c}$, and $\pi \Sigma_{c}$, which was developed in close analogy to the meson-exchange $\bar{K} N$ interaction of the Jülich group [14], utilizing $S U(4)$ symmetry constraints. The main ingredients of the $D N$ interaction are provided by vector meson $(\rho, \omega)$ exchange but higher-order box diagrams involving $D^{*} N, D \Delta$, and $D^{*} \Delta$ intermediate states, are also included. The coupling of the $D N$ system to the $\pi \Lambda_{c}$ and $\pi \Sigma_{c}$ channels is facilitated by $D^{*}(2010)$ exchange and by nucleon $u$-channel pole diagrams.

The interaction model generates several states dynamically. The narrow $D N$ quasi-bound state found in the $S_{01}$ partial wave is identified with the $(I=0)$ $\Lambda_{c}(2595)$-resonance. Narrow states were also found in the $S_{11}$ and $P_{01}$ partial waves. We identify the former with the $I=1$ resonance $\Sigma_{c}(2800)$ and the latter with the $\Lambda_{c}(2765)$-resonance, whose quantum numbers are not yet established [25].

Results for $D N$ total and differential cross sections were presented and compared with predictions of two 
interaction models that are based on the leading-order Weinberg-Tomozawa term using $S U(4)$ [28] or $S U(8)$ [30] symmetry. While the predictions of the Jülich and $S U(4)$ WT models for the $I=1$ channel are fairly similar, in magnitude as well as with regard to the energy dependence, this is not the case for the $I=0$ amplitude. Here the possible presence of a $P$-wave resonance near the threshold, i.e. the $\Lambda_{c}(2765)$, has a dramatic influence on the shape and the energy dependence of the cross section. The $S U(8)$ WT results are drastically different altogether, reflecting the different resonant structure of this interaction.

Finally, we discussed the $\Lambda_{c}(2595)$-resonance and the role of the near-by $\pi \Sigma_{c}$ threshold. In particular, we presented results for the $\pi \Sigma_{c}$ invariant-mass spectrum in the particle basis which illustrate the subtle effects of the slightly different thresholds of the $\pi^{+} \Sigma_{c}^{0}, \pi^{0} \Sigma_{c}^{+}$, and $\pi^{-} \Sigma_{c}^{++}$channels on the various invariant-mass distributions. We also pointed out that there seems to be a contradiction between the observation that the narrow $\Lambda_{c}(2595)$-resonance decays almost exclusively into the $\pi^{+} \Sigma_{c}^{0}$ and $\pi^{-} \Sigma_{c}^{++}$channels and the latest values of its mass, which place the resonance about $2 \mathrm{MeV}$ below the thresholds of those channels $[24,70]$. Indeed with a mass of $2592.06 \pm 0.3 \mathrm{MeV}$, as determined in ref. [70], the $\Lambda_{c}(2595)$ would lie just between the $\pi^{0} \Sigma_{c}^{+}$and the $\pi^{+} \Sigma_{c}^{0} / \pi^{-} \Sigma_{c}^{++}$ thresholds, which surely would be an interesting scenario.

J.H. acknowledges instructive discussions with M. Döring about the determination of poles in the complex plane. This work is partially supported by the Helmholtz Association through funds provided to the virtual institute "Spin and strong QCD" (VH-VI-231), by the EU Integrated Infrastructure Initiative HadronPhysics2 Project (WP4 QCDnet), by BMBF (06BN9006) and by DFG (SFB/TR 16, "Subnuclear Structure of Matter"). G.K. acknowledges financial support from CAPES, CNPq and FAPESP (Brazilian agencies). L.T. wishes to acknowledge support from the Rosalind Franklin Programme of the University of Groningen (The Netherlands) and the Helmholtz International Center for FAIR within the framework of the LOEWE program by the State of Hesse (Germany).

\section{Appendix A. The interaction Lagrangians}

In this appendix we list the specific interaction Lagrangians which are used to derived the meson-exchange $\bar{D} N$ interaction. The baryon-baryon-meson couplings are given by [14]

$$
\begin{aligned}
\mathcal{L}_{B B S}= & g_{B B S} \bar{\Psi}_{B}(x) \Psi_{B}(x) \Phi_{S}(x), \\
\mathcal{L}_{B B P}= & g_{B B P} \bar{\Psi}_{B}(x) i \gamma^{5} \Psi_{B}(x) \Phi_{P}(x), \\
\mathcal{L}_{B B V}= & g_{B B V} \bar{\Psi}_{B}(x) \gamma_{\mu} \Psi_{B}(x) \Phi_{V}^{\mu}(x) \\
& +\frac{f_{B B V}}{4 m_{N}} \bar{\Psi}_{B}(x) \sigma_{\mu \nu} \Psi_{B}(x)\left(\partial^{\mu} \Phi_{V}^{\nu}(x)-\partial^{\nu} \Phi_{V}^{\mu}(x)\right), \\
\mathcal{L}_{B \Delta P}= & \frac{f_{B \Delta P}}{m_{P}} \bar{\Psi}_{\Delta \mu}(x) \Psi_{B}(x) \partial^{\mu} \Phi_{P}(x)+H . c .
\end{aligned}
$$

$$
\begin{aligned}
\mathcal{L}_{B \Delta V}= & \frac{f_{B \Delta V}}{m_{V}} i\left(\bar{\Psi}_{\Delta \mu}(x) \gamma^{5} \gamma_{\mu} \Psi_{B}(x)\right. \\
& \left.-\bar{\Psi}_{B}(x) \gamma^{5} \gamma_{\mu} \Psi_{\Delta \mu}(x)\right)\left(\partial^{\mu} \Phi_{V}^{\nu}(x)-\partial^{\nu} \Phi_{V}^{\mu}(x)\right)
\end{aligned}
$$

Here, $\Psi_{B}$ and $\Psi_{\Delta \mu}$ are the nucleon (or hyperon) and $\Delta$ field operators and $\Phi_{S}, \Phi_{P}$, and $\Phi_{V}^{\mu}$ are the field operators for scalar, pseudoscalar and vector mesons, respectively.

The employed three-meson couplings are

$$
\begin{aligned}
\mathcal{L}_{P P S} & =g_{P P S} m_{P} \Phi_{P}(x) \Phi_{P}(x) \Phi_{S}(x), \\
\mathcal{L}_{P P V} & =g_{P P V} \Phi_{P}(x) \partial_{\mu} \Phi_{P}(x) \Phi_{V}^{\mu}(x), \\
\mathcal{L}_{V V P} & =\frac{g_{V V P}}{m_{V}} i \epsilon_{\mu \nu \tau \delta} \partial^{\mu} \Phi_{V}^{\nu}(x) \partial^{\tau} \Phi_{V}^{\delta}(x) \Phi_{P}(x),
\end{aligned}
$$

where $\epsilon_{\mu \nu \tau \delta}$ is the totally antisymmetric tensor in four dimensions with $\epsilon^{0123}=1$. Details on the derivation of the meson-baryon interaction potential from those Lagrangians can be found in ref. [14] together with explicit expressions for those potentials. The $S U(4)$ flavour structure that leads to the characteristic relations between the coupling constants is discussed in sect. II of [4]. All vertices are supplemented with form factors in order to suppress the meson-exchange contributions for high-momentum transfer and guarantee convergence when solving the Lippmann-Schwinger equation. For all ( $t$-channel) exchange diagrams those vertex form factors are parameterized in a conventional monopole form [14]

$$
F_{\alpha}\left(q^{2}\right)=\left(\frac{\Lambda_{\alpha}^{2}-m_{e x c h}^{2}}{\Lambda_{\alpha}^{2}+\mathbf{q}^{2}}\right)^{n_{\alpha}}
$$

where $\mathbf{q}^{2}$ is the square of the three-momentum transfer. Here $n_{\alpha}=1$ is used for all vertices except for the $N \Delta \rho$ vertex where $n_{\alpha}=2[14,75]$. In case of ( $s$ and $u$ channels) pole contributions a slightly different form is used to avoid problems of convergence and singularity, viz.

$$
F_{\beta}\left(q^{2}\right)=\left(\frac{\Lambda_{\beta}^{4}+m_{e x c h}^{4}}{\Lambda_{\beta}^{4}+\left(q^{2}\right)^{2}}\right),
$$

where $q^{2}$ is the square of the four-momentum transfer [14]. Note that both forms are normalized in such a way that $F \equiv 1$ when the exchanged particle is on its mass shell. The values for the vertex parameters (coupling constants and cut-off masses) that are used in our meson-exchange model of the $D N$ interaction are summarized for convenience in table 3 . The following averaged masses are used for evaluating the interaction potential and when solving the Lippmann-Schwinger equation in isospin basis: $M_{N}=$ $938.926 \mathrm{MeV}, M_{\Lambda_{c}}=2286.5 \mathrm{MeV}, M_{\Sigma_{c}}=2455.0 \mathrm{MeV}$, $M_{\pi}=138.03 \mathrm{MeV}, M_{D}=1866.9 \mathrm{MeV}$. For the calculation in the particle basis we use the masses as given in the PDG listing [25].

Open Access This article is distributed under the terms of the Creative Commons Attribution Noncommercial License which permits any noncommercial use, distribution, and reproduction in any medium, provided the original author(s) and source are credited. 
Table 3. Vertex parameters used in the meson-exchange model of the $D N$ interaction. $m_{\text {exch }}$ is the mass of the exchanged particle. $g_{M}$ and $g_{B} / f_{B}\left(\Lambda_{M}\right.$ and $\left.\Lambda_{B}\right)$ refer to the coupling constants (cut-off masses) used at the meson-meson-meson and baryon-baryon-meson (for pole and baryon-exchange graphs: upper and lower) vertex, respectively. The $\sigma$ and $a_{0}$ coupling constants in brackets are those that follow from our $\bar{D} N$ model [4] under the assumption of $S U(4)$ symmetry.

\begin{tabular}{|c|c|c|c|c|c|c|}
\hline Process & Exch. part. & $\begin{array}{l}m_{\text {exch }} \\
{[\mathrm{MeV}]}\end{array}$ & $g_{M} g_{B} /(4 \pi)$ & $g_{M} f_{B} /(4 \pi)$ & $\begin{array}{c}\Lambda_{M} \\
{[\mathrm{GeV}]}\end{array}$ & $\begin{array}{c}\Lambda_{B} \\
{[\mathrm{GeV}]}\end{array}$ \\
\hline \multirow[t]{6}{*}{$D N \rightarrow D N$} & $\rho$ & 769 & 0.773 & 4.713 & 1.4 & 1.6 \\
\hline & $\omega$ & 782.6 & -2.318 & 0.0 & 1.5 & 1.5 \\
\hline & $\sigma$ & 600 & $2.60[1.00]$ & - & 1.7 & 1.2 \\
\hline & $a_{0}$ & 980 & $-4.80[-2.60]$ & - & 1.5 & 1.5 \\
\hline & $\Lambda_{c}$ & 2286.5 & 15.55 & - & 1.4 & 1.4 \\
\hline & $\Sigma_{c}$ & 2455 & 0.576 & - & 1.4 & 1.4 \\
\hline \multirow[t]{2}{*}{$D N \rightarrow D^{*} N$} & $\pi$ & 138.03 & 3.197 & - & 1.3 & 0.8 \\
\hline & $\rho$ & 769 & -0.773 & -4.713 & 1.4 & 1.0 \\
\hline \multirow[t]{2}{*}{$D N \rightarrow D^{*} \Delta$} & $\pi$ & 138.03 & 0.506 & - & 1.2 & 0.8 \\
\hline & $\rho$ & 769 & -4.839 & - & 1.3 & 1.0 \\
\hline$D N \rightarrow D \Delta$ & $\rho$ & 769 & 4.839 & - & 1.3 & 1.6 \\
\hline \multirow[t]{3}{*}{$D N \rightarrow \pi \Lambda_{c}$} & $D^{*}$ & 2009 & -1.339 & -4.365 & 3.1 & 3.1 \\
\hline & $N$ & 938.926 & -14.967 & - & 2.5 & 1.4 \\
\hline & $\Sigma_{c}$ & 2455 & 1.995 & - & 3.5 & 1.4 \\
\hline \multirow[t]{4}{*}{$D N \rightarrow \pi \Sigma_{c}$} & $D^{*}$ & 2009 & -0.773 & 1.871 & 3.1 & 3.1 \\
\hline & $N$ & 938.926 & 2.88 & - & 2.5 & 1.4 \\
\hline & $\Lambda_{c}$ & 2286.5 & -10.368 & - & 2.8 & 1.4 \\
\hline & $\Sigma_{c}$ & 2455 & 2.304 & - & 3.5 & 1.4 \\
\hline$\pi \Lambda_{c} \rightarrow \pi \Lambda_{c}$ & $\Sigma_{c}$ & 2455 & 6.912 & - & 3.5 & 3.5 \\
\hline \multirow[t]{2}{*}{$\pi \Lambda_{c} \rightarrow \pi \Sigma_{c}$} & $\rho$ & 769 & 0.0 & 7.605 & 2.0 & 1.35 \\
\hline & $\Sigma_{c}$ & 2455 & 7.891 & - & 3.5 & 3.5 \\
\hline \multirow[t]{3}{*}{$\pi \Sigma_{c} \rightarrow \pi \Sigma_{c}$} & $\rho$ & 769 & 3.092 & 5.689 & 2.0 & 1.16 \\
\hline & $\Lambda_{c}$ & 2286.5 & 6.912 & - & 2.8 & 2.8 \\
\hline & $\Sigma_{c}$ & 2455 & 9.216 & - & 3.5 & 3.5 \\
\hline
\end{tabular}

\section{References}

1. PANDA Collaboration (I. Lehmann), arXiv:0909.4237 [hep-ex].

2. CBM Collaboration (P. Staszel), Acta Phys. Pol. B 41 $341(2010)$.

3. http://www.gsi.de/fair/.

4. J. Haidenbauer, G. Krein, U.-G. Meißner, A. Sibirtsev, Eur. Phys. J. A 33, 107 (2007).

5. J. Haidenbauer, G. Krein, U.-G. Meißner, A. Sibirtsev, Eur. Phys. J. A 37, 55 (2008).

6. T. Matsui, H. Satz, Phys. Lett. B 178, 416 (1986).

7. R.L. Thews, M. Schroedter, J. Rafelski, Phys. Rev. C 63, 054905 (2001).

8. K. Tsushima, D.H. Lu, A.W. Thomas, K. Saito, R.H. Landau, Phys. Rev. C 59, 2824 (1999).

9. C. Garcia-Recio, J. Nieves, L. Tolos, Phys. Lett. B 690, 369 (2010).

10. S.J. Brodsky, I.A. Schmidt, G.F. de Teramond, Phys. Rev. Lett. 64, 1011 (1990).

11. M.E. Luke, A.V. Manohar, M.J. Savage, Phys. Lett. B 288, 355 (1992).
12. G. Krein, A.W. Thomas, K. Tsushima, arXiv:1007.2220 [nucl-th] to be published in Phys. Lett. B.

13. S.H. Lee, C.M. Ko, Phys. Rev. C 67, 038202 (2003).

14. A. Müller-Groeling, K. Holinde, J. Speth, Nucl. Phys. A 513, 557 (1990).

15. R. Büttgen, K. Holinde, A. Müller-Groeling, J. Speth, P. Wyborny, Nucl. Phys. A 506, 586 (1990).

16. M. Hoffmann, J.W. Durso, K. Holinde, B.C. Pearce, J. Speth, Nucl. Phys. A 593, 341 (1995).

17. N. Isgur, M.B. Wise, Phys. Lett. B 232, 113 (1989).

18. M. Neubert, Phys. Rep. 245, 259 (1994).

19. R. Casalbuoni, A. Deandrea, N. Di Bartolomeo, R. Gatto, F. Feruglio, G. Nardulli, Phys. Rep. 281, 145 (1997).

20. W.A. Bardeen, E.J. Eichten, C.T. Hill, Phys. Rev. D 68, 054024 (2003).

21. K.W. Edwards et al., Phys. Rev. Lett. 74, 3331 (1995).

22. P.L. Frabetti et al., Phys. Lett. B 365, 461 (1996).

23. H. Albrecht et al., Phys. Lett. B 402, 207 (1997).

24. Jiu Zheng, PhD Thesis, University of Florida, 1999, http://www.lns. cornell.edu/public/THESIS/1999/ THESIS99-4/JiuZheng.ps. 
25. Particle Data Group (C. Amsler et al.), Phys. Lett. B 667, 1 (2008)

26. M.F.M. Lutz, E.E. Kolomeitsev, Nucl. Phys. A 730, 110 (2004).

27. J. Hofmann, M.F.M. Lutz, Nucl. Phys. A 763, 90 (2005).

28. T. Mizutani, A. Ramos, Phys. Rev. C 74, 065201 (2006).

29. M.F.M. Lutz, C.L. Korpa, Phys. Lett. B 633, 43 (2006).

30. C. Garcia-Recio, V.K. Magas, T. Mizutani, J. Nieves, A. Ramos, L.L. Salcedo, L. Tolos, Phys. Rev. D 79, 054004 (2009).

31. C.E. Jimenez-Tejero, A. Ramos, I. Vidana, Phys. Rev. C 80, 055206 (2009).

32. L. Tolos, J. Schaffner-Bielich, A. Mishra, Phys. Rev. C 70, $025203(2004)$.

33. L. Tolos, A. Ramos, T. Mizutani, Phys. Rev. C 77, 015207 (2008).

34. W. Weise, Nucl. Phys. A 835, 51 (2010).

35. D. Jido et al., Nucl. Phys. A 835, 59 (2010).

36. Y. Aikaishi, T. Yamazaki, M. Obu, M. Wada, Nucl. Phys. A 835, 67 (2010).

37. A. Gal, Chin. Phys. C 34, 1169 (2010) (arXiv:0912.2214 [nucl-th]).

38. D. Hadjimichef, J. Haidenbauer, G. Krein, Phys. Rev. C 66, 055214 (2002).

39. Y. Dong, A. Faessler, T. Gutsche, V.E. Lyubovitskij, Phys. Rev. D 81, 074011 (2010)

40. B. Borasoy, U.-G. Meißner, R. Nißler, Phys. Rev. C 74 055201 (2006).

41. U.-G. Meißner, U. Raha, A. Rusetsky, Eur. Phys. J. C 35, 349 (2004).

42. DEAR Collaboration (G. Beer et al.), Phys. Rev. Lett. 94, 212302 (2005).

43. DEAR Collaboration (M. Cargnelli et al.), Int. J. Mod. Phys. A 20, 341 (2005).

44. M. Iwasaki et al., Phys. Rev. Lett. 78, 3067 (1997).

45. T.M. Ito et al., Phys. Rev. C 58, 2366 (1998).

46. M. Cargnelli et al., Nucl. Phys. A 835, 27 (2010).

47. M. Cargnelli, in Proceedings of the 12th International Conference on Meson-Nucleon Physics and the Structure of the Nucleon May 31-June 4, 2010, MENU2010, AIP Conf. Proc., in preparation.

48. R.J. Nowak et al., Nucl. Phys. B 139, 61 (1978).

49. T.N. Tovee et al., Nucl. Phys. B 33, 493 (1971).

50. J.A. Oller, U.-G. Meißner, Phys. Lett. B 500, 263 (2001).
51. C. Garcia-Recio, J. Nieves, E. Ruiz Arriola, M.J. Vicente Vacas, Phys. Rev. D 67, 076009 (2003).

52. D. Jido, J.A. Oller, E. Oset, A. Ramos, U.-G. Meißner, Nucl. Phys. A 725, 181 (2003).

53. J.A. Oller, J. Prades, M. Verbeni, Phys. Rev. Lett. 95, 172502 (2005).

54. B. Borasoy, R. Nissler, W. Weise, Eur. Phys. J. A 25, 79 (2005).

55. J. Révai, N.V. Shevchenko, Phys. Rev. C 79, 035202 (2009).

56. E. Oset, A. Ramos, Nucl. Phys. A 635, 99 (1998).

57. R.J. Hemingway, Nucl. Phys. B 253, 742 (1985).

58. D.W. Thomas, A. Engler, H.E. Fisk, R.W. Kraemer, Nucl. Phys. B 56, 15 (1973).

59. D. Jido, E. Oset, T. Sekihara, Eur. Phys. J. A 42, 257 (2009).

60. J.C. Nacher, E. Oset, H. Toki, A. Ramos, Phys. Lett. B 455, 55 (1999).

61. S. Prakhov et al., Phys. Rev. C 70, 034605 (2004).

62. I. Zychor et al., Phys. Lett. B 660, 167 (2008).

63. V.K. Magas, E. Oset, A. Ramos, Phys. Rev. Lett. 95, 052301 (2005).

64. T. Hyodo, A. Hosaka, E. Oset, A. Ramos, M.J. Vicente Vacas, Phys. Rev. C 68, 065203 (2003).

65. R. Schumacher, Nucl. Phys. A 835, 231 (2010).

66. K. Moriya, R. Schumacher, Nucl. Phys. A 835, 325 (2010).

67. O. Krehl, C. Hanhart, S. Krewald, J. Speth, Phys. Rev. C 62, 025207 (2000).

68. A.M. Gasparyan, J. Haidenbauer, C. Hanhart, J. Speth, Phys. Rev. C 68, 045207 (2003).

69. L. Tolos, C. Garcia-Recio, J. Nieves, Phys. Rev. C 80, 065202 (2009).

70. A.E. Blechman, A.F. Falk, D. Pirjol, J.M. Yelton, Phys. Rev. D 67, 074033 (2003).

71. C. Hanhart, Yu.S. Kalashnikova, A.V. Nefediev, Phys. Rev. D 81, 094028 (2010).

72. V. Baru, J. Haidenbauer, C. Hanhart, Yu. Kalashnikova, A.E. Kudryavtsev, Phys. Lett. B 586, 53 (2004).

73. V. Baru, J. Haidenbauer, C. Hanhart, A.E. Kudryavtsev, U.-G. Meißner, Eur. Phys. J. A 23, 523 (2005).

74. E852 Collaboration (J. Gunter et al.), Phys. Rev. D 64, 072003 (2001).

75. R. Machleidt, K. Holinde, Ch. Elster, Phys. Rep. 149, 1 (1987). 